\title{
Die Oxydationszahlen des Harns in saurer und alkalischer Lösung.
}

\author{
Von
}

L. Niemilowiez und G. Gittelmacher-Wilenko.

Mit zwei Curventafeln.

(Aus dem medicinisch-chemischen Laboratorium der Universität Lemberg.)

(Der Redaction zugegangen am 14. Juli 1902.)

Die leicht ${ }^{1}$ ) reducirenden Körper des Harns, die in gewissen Grenzen der Ausdruck der im Blute circulirenden Körper sind und deshalb in physiologischen und pathologischen Processen Interesse bieten, könnte man in drei Gruppen theilen:

die Gruppe der Glucose, welche in alkalischer Lösung durch Oxyde der Schwermetalle in der Hitze leicht oxydirt, während sie in der Kälte durch das Chamäleon in saurer Lösung schwer angegriffen wird;

die Gruppe der Harnsäure, deren Verhalten gerade umgekehrt ist;

die Gruppe der Alkaptonsäuren, welche die Vortheile der ersten und zweiten Gruppe in sich vereinigen.

1) Als leicht reducirende Körper fasste ich diejenigen Körper auf, die in der Menge und Concentration von 0,1 Milligrammmolen in $100 \mathrm{ccm}$ die Fehling'sche Lösung unter der Siedehitze reduciren oder in saurer Lösung die Indigocarminoxydationszahl strecken. - Die erstere Definition wurde nicht in den Kreis der vorliegenden Untersuchungen einbezogen. 
Die bisher üblichen Methoden der Bestimmung der reducirenden Körper des Harns entsprechen auch mehr der einen oder der anderen Gruppe dieser Körper. Es sind dies die Oxydationsmethoden mit Fehling'scher, Pavy'scher und Knapp'scher Lösung, deren sich Flückiger, E. Salkowski, Munck, Moritz, Worm-Müller, Hagemann, St. Jonson, v. Udránszky, Pavy und Andere bedient haben. Dann die Methode von Haeckenhayn ${ }^{1}$ ) mit Orthonitrophenylpropiolsäure und Natronlauge. Die Methode von Rosin, ${ }^{2}$ ) wo ebenfalls in alkalischer Lösung in der Hitze mit Permanganat so lange oxydirt wird, bis die übrig bleibenden reducirenden Körper das Methylenblau nicht mehr reduciren können. Dann die Methode von Byasson, ${ }^{3}$ ) wo in der Kälte und in schwefelsaurer Lösung mit überschüssigem Permanganat gearbeitet wird; endlich die Methode von Etard und Richet, $\left.{ }^{+}\right)$wo als Oxydationsmittel Bromwasser in saurer Lösung dient.

Eine neue Methode zur quantitativen Prüfung der reducirenden Wirkung des Harns wurde unlängst von einem von uns ${ }^{5}$ ) angegeben.

Dieselbe bestimmt die reducirenden Körper des Harns mit Ausschluss der Zuckergruppe, und von den übrigen nur diejenigen, welche am stärksten reducirend wirken und diesbezüglich auf der Stufe der Harnsäure stehen. Es werden durch diese Methode alle Körper bestimmt, welche leichter als die Harnsäure reduciren, alle welche diesbezüglich mit der Harnsäure gleichwerthig sind, die Harnsäure selbst quantitativ und von den anderen Körpern nur theilweise diejenigen, welche der Harnsäure in Bezug auf die reducirende Wirkung am nächsten stehen und quantitativ reichlich vorkommen.

Andere Körper, auch diejenigen, welche durch Chamäleonüberschüsse leicht oxydirt werden (wie bei der Methode von Byasson), kommen da nicht in Betracht.

1) Dissertation, Erlangen, 1887.

2) Chemisches Centralblatt, 1900, Bd. 1, S. 48.

3) Siehe aus Meillerc, Compt. red. soc. biolog., Bd. 52, S. 325.

4) J. Th., Bd. 12, S. 184; Bd. 13, S. 195.

5) L. Niemilowicz, Diese Zeitschrift, Bd. XXXV, S. 264. 
Ausser dieser Methode der Oxydation in saurer Lösung führen wir eine zweite Methode der Oxydation in alkalischer Lösung ein, wobei auch die Harnsäure als Grenzkörper dient. Als Oxydationsmittel verwenden wir 1/50-Normal-Ferricyankaliumlösung, als Indicator das Alizarin in 0,1\% iger alkoholischer Lösung.

Durch diese Methoden wurden auch einige Körper der Harnsäure und Alkaptonsäurengruppe ermittelt, die Körper der Zuckergruppe nicht bestimmt.

Vor den bisherigen Methoden haben die neuen Einiges voraus. Einmal deshalb, weil durch das beschriebene Verfahren, die durch ihre reducirende Wirkung am meisten aktiven, möglicher Weise leicht zu Autointoxicationen führenden Körper quantitativ bestimmt werden und zweitens, weil die Methode eine Handhabe gibt, um nach diesen grösstentheils unbekannten Körpern weiter $z u$ forschen. Es können nämlich nur solche Körper in Frage kommen, welche Indigocarmin, respective Alizarinoxydationszahl strecken und das ist, wie weiter beschrieben wird, leicht zu prüfen.

Aus diesem Grunde haben wir auch weiterhin die in normalen Verhältnissen vorkommenden Beziehungen ermittelt und einige Beispiele angegeben, wie man bei pathologischen Harnen verfahren soll.

\section{Die Oxydationsmethode in saurer Lösung. ${ }^{1}$ )}

Nach dem am angeführten Orte beschriebenen Verfahren bestimmt man die Nettooxydationszahl NTO\% (für $100 \mathrm{ccm}$ Harn). Dann bestimmt man die Purinkörper nach Denigès und die Xanthinkörper nach der OF-Methode $^{1}$ ) und ermittelt auf diese Art die Harnsäure, was die beste und leichteste quantitative Methode der Harnsäurebestimmung im Harne ist. ${ }^{2}$ ) Die Menge der Harnsäure in Milligrammen wird durch 7,4 dividirt und man bekommt auf diese Weise denjenigen Theil

1) Niemilowicz, 1. c.

2) G. Gittelm a cher-Wilenk o, Diese Zeitschrift, Bd. XXXVI, S. 21. 
der NTO'-Zahl, die der Harnsäure entspricht. Wir nennen diese Zahl UTO' und die Differenz NTO'-UTO' nennen wir RTO'-Restoxydationszahl und bestimmen damit diejenige Menge 1/10-Normal-Chamäleonlösung, die für die Oxydation der anderen reducirenden Körper der Harnsäurestufe nothwendig sind.

Nachdem diese Zahlen in saurer Lösung erhalten wurden, zeichnen wir sie mit ', zum Unterschiede von den analogen Zahlen der alkalischen Methode, die wir mit - bezeichnen. Alle diese Zahlen berechnen wir auf die ganze untersuchte Menge des Harns.

\section{Die Oxydationsmethode in alkalischer Lösung.}

Man gibt in einen Evacuirkolben mit Seitentubus $100 \mathrm{ccm}$ Harn und schliesst die Flasche mit einem Kautschukstöpsel mit 2 Bohrungen. In einer Bohrung steckt der beinahe capillar ausgezogene Ablauf einer graduirten Bürette mit 1/50-NormalFerricyankaliumlösung, in der zweiten ein ebenfalls beinahe capillar ausgezogenes Röhrchen, welches an dem Luftende mit Schlauch und Federklemme versehen ist.

Man evacuirt den Kolben und dann setzt man durch den Schlauch $5 \mathrm{ccm} 30 \%$ ige Natronlauge; darauf langsam, tropfenweise, unter schnellem Umschwenken $15 \mathrm{ccm} 1 / \mathbf{5}_{01-}$ Normal-Ferricyankaliumlösung. Dann öffnet man den Kolben, filtrirt $100 \mathrm{ccm}$ vollkommen klar ab, gibt dieselben in ein Becherglas mit $7 \mathrm{~cm}$ lichter Weite, setzt $1 \mathrm{ccm} 0,1 \%$ ige Alizarinlösung hinzu und titrirt mit 1/50-Normal-Ferricyankaliumlösung (aus einer anderen Bürette) so lange, bis die 2 Absorbtionsstreifen des alkalischen Alizarins in roth und gelb gerade verschwunden sind.

Man setzt wieder $1 \mathrm{ccm}$ Alizarinlösung hinzu, titrirt auf dieselbe Art und wiederholt das noch einmal.

Man überzeugt sich, dass bei richtiger Ausführung der Analyse die gleiche Strecke bereits nach der ersten Oxydation erreicht wurde, so dass man die zweite und dritte Theilstrecke nicht der BTO-Zahl zuzählen darf.

Man wiederholt die Bestimmung mit einem Harn, der 
bei der Nachoxydation etwa $12 \mathrm{ccm}$ 1/50-Normal-Ferricyankalium braucht, indem man den ursprünglichen Harn entsprechend verdünnt und nimmt diese Zahl zur Basis der Berechnung.

Die Berechnung der NTO-Zahl führt man foldendermaassen aus. Von den $15 \mathrm{ccm}$ der zuerst zugesetzten Ferricyankaliumlösung sind im Filtrat $12,5 \mathrm{ccm}$ vorhanden, dazu kommt die zum zweiten Male zugesetzte Menge und die Summe ist BT0. Von dieser Bruttooxydationszahl zieht man die Alizarinoxydationszahl $(0,8)$ ab und dann ist BTO $-0,8 \cdot \frac{6}{5}=$ $\frac{\mathrm{NTO}}{5} \%$.

NTO $\%$ ist diejenige Menge $1 / 10$-Normal-Ferricyankaliumlösung, welche nothwendig ist, um $100 \mathrm{ccm}$ Harn mit Alizarin als Indicator auf gleiche Strecke $\mathrm{zu}$ oxydiren, und NTO ist dieselbe Zahl auf die ganze Harnmenge bezogen. ${ }^{1}$ )

Um UTO und RTO zu bestimmen, muss man das Verhältniss zwischen der Harnsäure und dem Ferricyankalium kennen. $1 \mathrm{ccm} 1 / 10$-Normal-Ferricyankalium entspricht unter den gegebenen Verhältnissen 9,5 Milligrammen Harnsäure.

Die Harnsäuremenge in Milligrammen wird also mit 9,5 dividirt und der Quotient ist UTO; die Differenz NTO-UTO. ist $\mathrm{RTO} \cdot{ }^{2}$ )

1) Es kommen auch Harne vor, die zur Nachoxydation weniger als $12 \mathrm{ccm}$ brauchen. Fällt die nothwendige Menge nicht unter $5 \mathrm{ccm}$, so sind die Oxydationszahlen der Oxydirbarkeit des Harns proportional; sollten aber zur Nachoxydation weniger als $5 \mathrm{ccm}$ verwendet werden, so setzt man im Vacuo weniger als $15 \mathrm{ccm}$ hinzu und macht die entsprechende Umrechnung. Hat man z. B. im Vacuo 12 oder $9 \mathrm{ccm}$ hinzugesetzt, so ergänzt man zuerst das Volum des Reactionsproductes mit Wasser auf $120 \mathrm{ccm}$. (durch Zusatz von 3 resp. $6 \mathrm{ccm} \mathrm{H}_{2} \mathrm{O}$ ) und filtrirt wie sonst $100 \mathrm{ccm} \mathrm{ab}$, auf welche dann 10 resp. 7,5 ccm Ferricyankalium entfallen.

Die sonstige Verrechnung ist der im Texte angeführten gleich.

Die im Vacuo und die zur Nachoxydation verwendeten Mengen Ferricyankaliumlösung sollen annähernd gleich sein.

2) Die in der vorliegenden Arbeit angeführten alkalischen Oxyda- 
Die Erklärung der alkalischen Methode ist dieselbe wie die bei der sauren Oxydation, nur in dreifacher Hinsicht ist ein Unterschied zu verzeichnen:

1. Man versetzt den Harn mit Alkali und der Hälfte Ferricyankalium im Vacuo, um die Einwirkung der Luft auf die am leichtesten oxydirbaren Körper auszuschliessen.

Die noch übrig bleibenden reducirenden Körper werden dann beim Filtriren nicht mehr von der Luft oxydirt und man titrirt mit Ferricyankalium im offenen Gefässe zu Ende.

2. Alizarin darf nur in ganz klare Lösung gegeben werden, da es sich sonst auf den Phosphaten lackartig niederschlägt und nicht mehr leicht oxydirt wird.

3. Die zweite und dritte Oxydationstheilstrecke dienen nur zur Kontrolle der ersten. Sie dürfen nicht mehr als um $0,1 \mathrm{ccm}$ differiren und werden dann nicht BTO zugezählt.

Die grösstentheils unbekannten Körper, denen die RTOZahlen entsprechen, nennen wir die Restkörper.

tionszahlen der Harne wurden etwas anders ermittelt, indem mit 1/20Normal-Ferricyankalium titrirt wurde.

Wir nahmen $100 \mathrm{ccm}$ Harn, versetzten ihn mit $4,530 \%$ iger Natronlauge und tropfenweise mit $5,5 \mathrm{ccm} 1 / 20-$ Normal-Ferricyankaliumlösung. Davon filtrirten wir $100 \mathrm{ccm} \mathrm{ab}$, setzten Alizarin hinzu und titrirten auf gleiche Strecke bis die Absorbtionsstreifen in $\tilde{5} \mathbf{c m}$ dicker Schicht verschwanden.

Die Berechnung war folgende: $\mathrm{Zu} 5 \mathrm{ccm}$ 1/20-Normal-Ferricyankalium, welche im Filtrate vorhanden waren, setzten wir a Cubikcentimeter nach, somit brauchten wir für $100 \mathrm{ccm}$ des ursprünglichen Harns $5+a \cdot \frac{11}{10} \mathrm{ccm}$ 1/80-Normal-Ferricyankaliumlösung und die Hälfte davon ergab NTO $\%$, welche Zahl dann als NTO auf die ganze Harnmenge bezogen wurde.

Die alkalischen Oxydationszahlen, welche wir mit 1/20-NormalFerricyankalium bekamen, waren bei vorsichtiger Ausführung und genügender Concentration des Harns an Restkörpern denjenigen gleich, die mit 1/50-Normal-Ferricyankaliumlösung erhalten wurden. 


\section{Prüfung,}

ob eine Substanz zu den Restkörpern gezählt werden könnte.

Sie bietet ein Interesse für die weitere Forschung nach diesen Körpern und für das Verständniss, welche Bestandtheile des Harns gegenwärtig diesen Körpern zugezählt werden können.

Sie beruht darauf, dass man prüft, ob der untersuchte Körper die saure oder alkalische UTO-Zahl streckt.

2,688 g Harnsäure werden in $300 \mathrm{ccm}^{1 / 4}$-Normalnatronlauge in der Kälte gelöst, auf 1 Liter aufgefüllt und sofort verwendet.

Davon enthalten $25 \mathrm{ccm} 0,4 \mathrm{mg}$ Mole Harnsäure = $0,0672 \mathrm{~g}$.

Zur Prüfung in der sauren Lösung nimmt man $12,5 \mathrm{ccm}$ mit $0,2 \mathrm{mg}$ Molen (um beim Ansäuern keine Harnsäure auszufällen - für die alkalische Methode kann man $25 \mathrm{ccm}$ mit $0,4 \mathrm{mg}$ Molen nehmen) und füllt auf $100 \mathrm{ccm}$ auf, was der gewöhnlichen Concentration der Harnsäure im Harn entspricht.

In diesen $100 \mathrm{ccm}$ bestimmt man die NTO-Zahl, welche hier der UTO-Zahl gleich ist.

Eine zweite Probe mit derselben Menge Harnsäure in $50 \mathrm{ccm}$ versetzt man mit $50 \mathrm{ccm}$ einer Lösung von 0,2 Milligrammmolen der untersuchten Substanz und bestimmt wieder NTO' respective NTO. Wird eine derselben gegen früher gestreckt, so kann der untersuchte Körper ein Restkörper seị und man findet durch das Verhältniss der UTO- zu der RTOZahl wie gross die reducirende Kraft des Restkörpers im Vergleiche zur aequimolekularen Menge der Harnsäure ist.

Durch die Beobachtung der gleichen Strecke findet man auch, ob der Restkörper vollkommen oxydirt wird, denn dann ist die gleiche Strecke annähernd der Reactionsspanne des Indicators gleich, oder ob die verminderte Concentration des Restkörpers denselben oder seine ersten Oxydationsproducte auf annähernd gleiche Reactionsgeschwindigkeit mit dem Indicator stellt, denn dann wird die gleiche Strecke verlängert. 
Nach dieser Methode haben wir einige Körper geprüft und fanden:

Oxydation in saurer Lösung

$3 \mathrm{ccm} \mathrm{IC}=1 \mathrm{ccm}{ }^{1 / 10-N o r m a l c h a m a ̈ l e o n, ~}$

$7,4 \mathrm{mg}$ Harnsäure $=1 \mathrm{ccm}{ }^{1 / 10}$-Normalchamäleon.

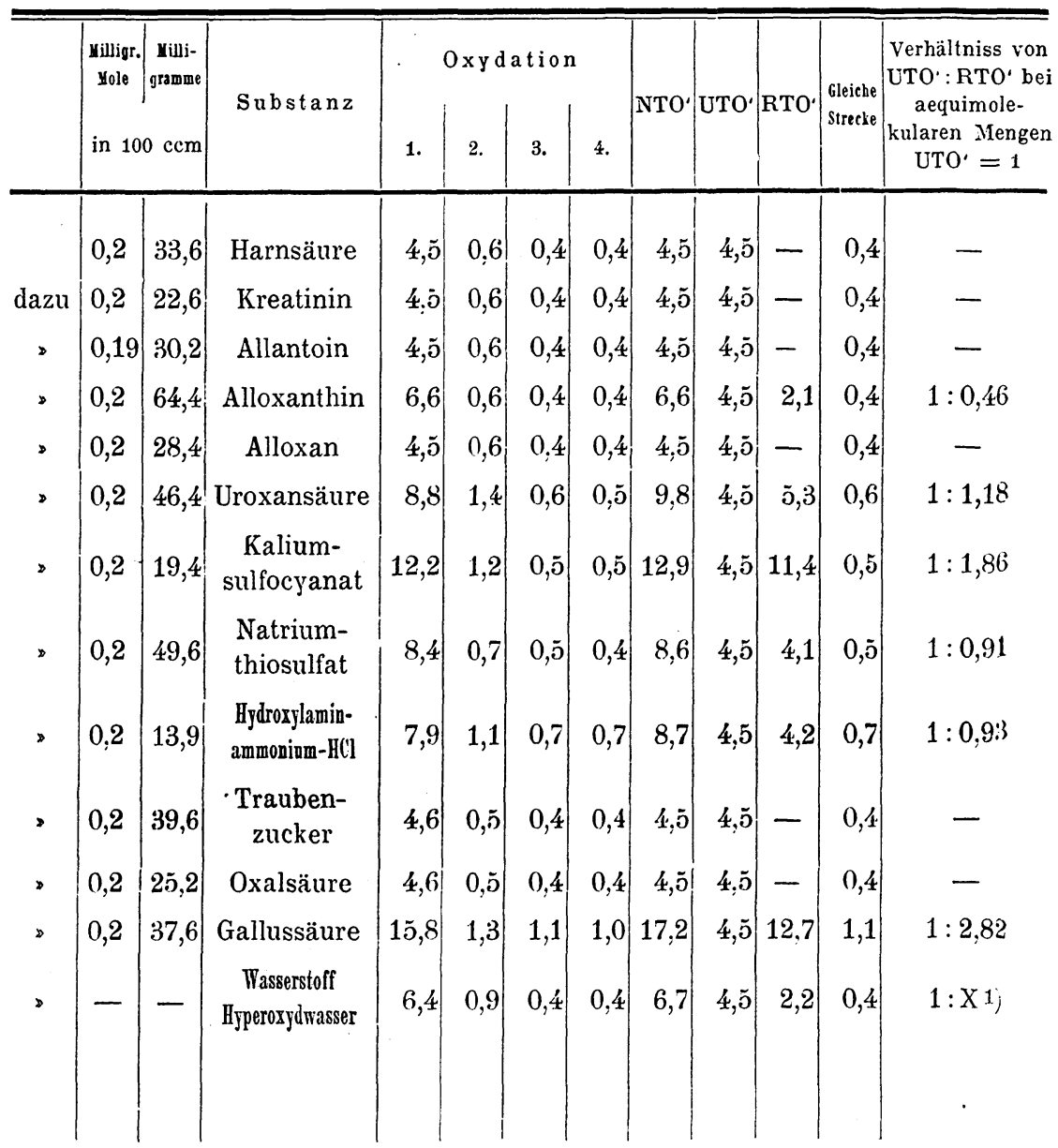

1) Wir beabsichtigten, durch die obigen Bestimmungen vorläufig nur qualitativ die Reaction zu prüfen. 


\section{Oxydation in alkalischer Lösung}

1 . ccm Alizarin $=0,8 \mathrm{~cm} 1 / 50$-Normalferricyankalium, $9,5 \mathrm{mg}$ Harnsäure $=1 \mathrm{ccm}{ }^{1 / 10}$-Normalferricyankalium.

\begin{tabular}{|c|c|c|c|c|c|c|c|c|c|c|c|c|}
\hline & villgr. & yilli- & & & $0 x y d$ & atios & & & & & & Verhältniss von \\
\hline & 0,4 & 67,2 & Harnsäure & 36,2 & 1,1 & 1,0 & 1,0 & 7,1 & 7,1 & - & 1,1 & - \\
\hline dæzu & 0,2 & 22,6 & Kreatinin & 36,2 & & & tre & $\operatorname{cktr}$ & nich & & & - \\
\hline 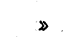 & 0,19 & 30,2 & Allantoin & 36,2 & & & » & & $\gg$ & & & - \\
\hline 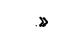 & 0,2 & 64,4 & Alloxanthin & 36,2 & & & » & & $»$ & & & - \\
\hline$D$ & 0,2 & 28,4 & Alloxan & 36,2 & & & » & & » & & & - \\
\hline$\gg$ & 0,2 & 46,4 & Uroxansäure & $60, \tilde{\check{s}}$ & 1,3 & 1,2 & 1,2 & $\mid 12,0$ & | $7,1 \mid$ & 4,9 & 1,3 & $1: 1,38$ \\
\hline » & 0,2 & 19,4 & $\begin{array}{c}\text { Kalium- } \\
\text { sulfocyanat }\end{array}$ & 36,2 & 1,3 & & tre & ckt r & $\mathrm{nich}$ & & & - \\
\hline$\curvearrowright$ & 0,2 & 49,6 & $\begin{array}{l}\text { Natrium- } \\
\text { thiosulfat }\end{array}$ & 36,2 & & & 》 & & 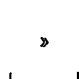 & & & - \\
\hline$\therefore$ & 0,2 & 13,9 & $\begin{array}{l}\text { Hydroxylamin- } \\
\text { ammonium-HCl }\end{array}$ & 51,5 & 1,1 & 1,1 & 1,1 & 10,2 & 7,1 & 3,1 & 1,1 & $1: 0,88$ \\
\hline$»$ & 0,2 & 39,6 & $\begin{array}{l}\text { Trauben- } \\
\text { zucker }\end{array}$ & 36,2 & 1,1 & & $\operatorname{tre}$ & $\mathrm{ckt}$ & $\mathrm{nich}$ & & & - \\
\hline$\triangleright$ & 0,2 & 25,2 & Oxalsäure & 36,2 & & & 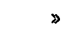 & & ه & & & - \\
\hline 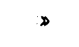 & 0,2 & 37,6 & Gallussäure & 59,4 & 1,9 & 1,9 & 1,6 & 11,7 & $7 \mid 7,1$ & 4,6 & 1,9 & $1: 1,29$ \\
\hline s & - & - & $\begin{array}{l}\text { Wasserrtofif } \\
\text { Hyperosydwasser }\end{array}$ & 36,2 & 1,2 & 1,3 & 1,4 & 7,1 & 7,1 & - & $\mid \begin{array}{c}1,2 \\
\text { stelgend }\end{array}$ & - \\
\hline
\end{tabular}

Aus dieser Tabelle sieht man:

1. dass Kreatinin, Allantoin, Alloxan, Traubenzucker, Oxalsäure die Harnsäure-Oxydationszahl weder in saurer mit Chamäleon, noch in alkalischer Lösung mit Ferricyankalium strecken ;

2. dass das Alloxanthin, Uroxansäure, Rhodankalium, Natriumthiosulfat, Hydroxylaminammonium-HCl, Wasserstoffhyperoxyd in saurer Lösung die UTO'-Zahl strecken;

3. dass die alkalische UTO-Zahl mit Ferricyankalium nur von einigen von diesen Körpern und zwar von: Uroxansäure, Hydroxylaminammonium $\mathrm{HCl}$ und Gallussäure gestreckt wird. 
4. dass die gleiche Strecke bedeutend vergrössert wird in saurer Lösung durch: Alloxanthin, Hydroxylaminammonium$\mathrm{HCl}$, Gallussäure; in alkalischer Lösung durch: Uroxansäure, Gallussäure, Wasserstoffhyperoxyd.

5. Wasserstoffhyperoxyd bewirkt in alkalischer Lösung mit Ferricyankalium, ohne bei der ersten Oxydation als Streckekörper zu wirken, eine Steigung der gleichen Strecke, was wir auf chemische Induction zurückführen.

Das Chamäleon oxydirt die Restkörper prompter und vollständiger als Ferricyankalium, was in der Verlängerung der gleichen Strecke mit dem letzteren Oxydationsmittel zum Ausdruck kommt. In Folge dessen sind die Schwankungen bei den alkalischen Oxydationszahlen des Harns viel geringer und weniger ausgeprägt als bei den sauren Oxydationszahlen desselben Harns. Jedenfalls sieht man aus diesen Zahlen, dass von den normalen Bestandtheilen des Harns das Rhodankalium zu den sauren Restkörpern gehört.

In Nachstehendem bringen wir die nach den obigen Methoden ausgeführten Untersuchungen zur Feststellung der Normalzahlen des physiologischen Harns.

Die Zahlen, die wir anführen, beziehen sich immer auf die ganze Harnmenge in der beobachteten Periode, also zuerst in der Tagesmenge und dann in der ersten und in der zweiten Verdauungsperiode, und sind Folgende:

1. $\mathrm{V}=$ Harnvolumen;

2. $\mathrm{H}=$ Harnsäure in Grammen;

3. $\mathrm{X}=$ Xanthinkörper in Grammen;

4. $N=$ Stickstoff in Grammen;

5. $\mathrm{NTO}^{\prime}=$ Nettooxydationszahl in saurer Lösung in Cubikcentimetern $1 / 10$-Normal-Chamäleon ausgedrückt.

6. UTO' = Harnsäureoxydationszahl in saurer Lösung in Cubikcentimetern 1/10-Normal-Chamäleon ausgedrückt;

7. $\mathrm{RTO}^{\prime}=$ Restkörperoxydationszahl in saurer Lösung in Cubikcentimetern 1/10-Normal-Chamäleon ausgedrückt;

8. NTO = Nettooxydationszahl in alkalischer Lösung in Cubikcentimetern $1 / 10$-Normal-Ferricyankalium ausgedrückt;

9. UTO $^{-}=$Harnsäureoxydationszahl in alkalischer Lösung in Cubikcentimetern 1/10-Normal-Ferricyankalium ausgedrückt; 
10. RTO $=$ Restkörperoxydationszahl in alkalischer Lösung in Cubikcentimetern 1/10-Normal-Ferricyankalium ausgedrückt;

11. auf $100 \mathrm{NTO}^{\prime}$ entfallen $\mathrm{UTO}^{\prime}$ und $\mathrm{RTO}^{\prime}$;

12. auf $100 \mathrm{NTO}^{*}$ entfallen UTO ${ }^{*}$ und RTO

13. $\frac{\mathrm{NTO}^{\prime}}{\mathrm{N}}\left\{\begin{array}{c}\text { Diese Werthe bezeichnen wir als den kalorischen } \\ \text { Verlust an sauren, respective alkalischen Restkörpern }\end{array}\right.$

14. $\frac{\text { NTO }}{N} \int \begin{aligned} & \text { sammt Harnsäure im Vergleiche mit dem Gesammt- } \\ & \text { stickstoffe des Harns. }\end{aligned}$

Zur Bestimmung dieser 14 Zahlen sind nun 5 Analysen nöthig, und zwar die Bestimmung des Stickstoffs nach Kjeldahl, der Xanthinkörper nach der von einem von uns angegebenen OF-Methode, der Harnsäure durch Kombination der letzteren mit der Methode von Denigès, der sauren NTO'-Zahl und der alkalischen NTO-Zahl. Alles andere ist Berechnung. Die nothwendigen Doppelbestimmungen an einem Harn können bequem in 2-3 Stunden ausgeführt werden.

\section{Bestimmung der Oxydationszahlen in normalen Harnen.}

Reihe I. Bei gemischter gleichmässiger Nahrung ohne Stickstoffgleichgewicht. Bei 4 Versuchspersonen.

Harn Z., 21 Jahre alt. 65 Kilo.

\begin{tabular}{|c|c|c|c|c|c|c|c|c|}
\hline \multirow[b]{2}{*}{ Tag } & \multirow{2}{*}{$\begin{array}{c}\text { Tages- } \\
\text { Menge } \\
\text { ccm }\end{array}$} & \multicolumn{5}{|c|}{ Mittelzahlen } & \multicolumn{2}{|c|}{$\mathrm{NTO}^{\prime}=100$} \\
\hline & & Harnsäure & $\begin{array}{l}\text { Xanthin. } \\
\text { körper }\end{array}$ & NTO & UTO' & RTO' & UTO' & RTO \\
\hline $6 . / \mathrm{V}$. & 1190 & 0,822 & $0,0: 376$ & 163,6 & 110,7 & 52,9 & 67,6 & 32,4 \\
\hline 7./V. & 1300 & 0,939 & 0,0346 & 187,2 & 126,9 & 60,3 & 67,7 & 32,3 \\
\hline 8./V. & 960 & 0,661 & 0,0420 & 137,3 & 89,3 & 48,0 & 65,0 & $3 \check{\jmath}, 0$ \\
\hline 10./V. & 820 & 0,591 & 0,0410 & 135,30 & 79,5 & 55,8 & 58,8 & 41,2 \\
\hline 13./V. & 1450 & 0,986 & 0,0744 & 182,7 & 133,4 & 49,3 & 72,9 & 27,1 \\
\hline 14./V. & 1100 & 0,917 & 0,0744 & 187,0 & 136,4 & 50,6 & 72,9 & 27,1 \\
\hline 15./V. & 910 & $0,74 \check{~}$ & 0,0493 & 150,2 & 100,2 & 50,0 & 66,6 & 33,4 \\
\hline \multicolumn{4}{|c|}{ Im Mittel. . . } & 163,3 & 110,9 & 52,4 & 67,4 & 32,6 \\
\hline
\end{tabular}


Harn G., 25 Jahre alt. 59 Kilo.

\begin{tabular}{|c|c|c|c|c|c|c|c|c|}
\hline \multirow[b]{2}{*}{ Tag } & \multirow[b]{2}{*}{$\begin{array}{c}\text { Tages- } \\
\text { Menge } \\
\text { ccm }\end{array}$} & \multicolumn{5}{|c|}{ Mittelzahlen } & \multicolumn{2}{|c|}{$\mathrm{NTO}^{\prime}=100$} \\
\hline & & Harnsäure & $\begin{array}{l}\text { Xanthin- } \\
\text { körper }\end{array}$ & NTO' & UTO' & $\mathrm{RTO}^{\prime}$ & UTO' & RTO' \\
\hline 6./V. & 1280 & $0,60 \check{~}$ & 0,032 & 147,8 & 81,6 & 66,2 & 55,2 & 44,8 \\
\hline 8./V. & 1300 & $(0,746$ & 0,033 & 178,1 & 101,0 & 77,1 & 56,7 & 43,3 \\
\hline 12./V. & 1010 & $0,58: 3$ & 0,041 & 138,4 & 68,7 & 69,7 & 57,0 & 43,0 \\
\hline 13./V. & 1600 & 0,622 & 0,030 & 148,0 & 83,8 & 64,2 & $56, \tilde{0}$ & 43,5 \\
\hline 14./V. & 10500 & 0,653 & 0,033 & 143,8 & 88,1 & 55,7 & 61,3 & 38,7 \\
\hline 15./V. & 1280 & 0,661 & 0,047 & 153,6 & 89,6 & 64,0 & 58,5 & 41,5 \\
\hline & & & & 151,6 & $8 \check{5}, 5$ & 66,1 & 57,5 & 42,5 \\
\hline
\end{tabular}

Harn T., 26 Jahre alt. 61 Kilo.

\begin{tabular}{|c|c|c|c|c|c|c|c|c|}
\hline \multirow[b]{2}{*}{ Tag } & \multirow{2}{*}{$\begin{array}{c}\text { Tages- } \\
\text { Menge } \\
\text { ccm }\end{array}$} & \multicolumn{5}{|c|}{ Mittelzahlen } & \multicolumn{2}{|c|}{$\mathrm{NTO}^{\prime}=100$} \\
\hline & & Ilarnsäure & $\begin{array}{c}\text { Xanthin- } \\
\text { körper }\end{array}$ & NTO' & UTO' & $\mathrm{RTO}^{\prime}$ & UTO' & RTO \\
\hline 8./V. & 1380 & 0,551 & 0,014 & 134,6 & 74,6 & 60,0 & $5 \check{5}, 4$ & 44,6 \\
\hline 13./V. & 980 & 0,836 & 0,054 & 212,7 & 125,5 & 87,2 & 59,0 & 41,0 \\
\hline $14 . / \mathrm{V}$. & 1520 & 0,699 & 0,058 & 168,0 & 94,3 & 73,7 & 56,1 & 43,9 \\
\hline 15./V. & 1260 & 0,508 & 0,036 & 119,7 & 68,3 & 51,4 & 57,6 & 43,0 \\
\hline & & & & 158,7 & 90,7 & 68,0 & 56,9 & 43,1 \\
\hline
\end{tabular}

Harn A., 30 Jahre alt. 49 Kilo.

\begin{tabular}{|c|c|c|c|c|c|c|c|c|}
\hline \multirow[b]{2}{*}{ Tag } & \multirow{2}{*}{$\begin{array}{c}\text { Tages- } \\
\text { menge } \\
\mathrm{ccm}\end{array}$} & \multicolumn{5}{|c|}{ Mittelzahlen } & \multicolumn{2}{|c|}{$\mathrm{NTO}^{\prime}=100$} \\
\hline & & Harnsäure & $\begin{array}{c}\text { Xanthin. } \\
\text { körper }\end{array}$ & NTO & UTO' & RTO & UTO' & RTO \\
\hline 7./V. & 1820 & 0,723 & 0,0237 & 171,1 & 98,5 & 72,6 & 57,3 & 42,7 \\
\hline 8.jV. & 2020 & 0,707 & 0,0345 & 185,8 & 96,5 & 89,3 & 51,7 & 48,3 \\
\hline $10 . / \mathrm{V}$ & 1420 & 0,775 & $0,0 \check{5} 40$ & 187,4 & 104,8 & 82,6 & 55,9 & 44,1 \\
\hline & & Mittel & • & 181,4 & 99,9 & 81,5 & $5 \check{5}, 0$ & 45,0 \\
\hline
\end{tabular}


Die Oxydationszahlen des Harns in saurer u. alkalischer Lösung. 179

Reihe II. Oxydationszahlen in der Tagesmenge bei gemischter, gleichmässiger, in denselben Zeiten genossener Nahrung und Stickstoffgleichgewicht. - Bei 2 Personen.

$$
\text { G., } 25 \text { Jahre alt. }
$$

\begin{tabular}{|c|c|c|c|c|c|c|c|c|c|c|}
\hline \multirow{3}{*}{ Tag } & \multirow{3}{*}{$\begin{array}{l}\text { Volum } \\
\mathrm{ccm}\end{array}$} & \multicolumn{9}{|c|}{ Mittelzahlen } \\
\hline & & \multirow{2}{*}{$\begin{array}{c}\mathrm{H} \\
\text { (Harn- } \\
\text { säure) }\end{array}$} & \multirow{2}{*}{$\underset{\text { (Xörper) }}{\mathrm{X}}$} & \multirow{2}{*}{$\mathbf{N}$} & \multirow{2}{*}{ NTO } & \multirow{2}{*}{ UTO } & \multirow{2}{*}{ RTO } & \multicolumn{2}{|c|}{$\mathrm{NTO}^{\prime}=100$} & \multirow{2}{*}{$\frac{\text { NTO }^{\prime}}{\mathrm{N}}$} \\
\hline & & & & & & & & UTO & RTO' & \\
\hline 7./IV. & 1645 & 1,057 & 0,038 & 22,0 & 208,9 & 141,5 & 67,4 & 67,7 & 32,3 & 9,5 \\
\hline $8 . / \mathrm{IV}$. & 1190 & $0,97 \check{5}$ & 0,029 & 22,3 & 204,7 & 132,1 & 72,6 & 64,5 & 35,5 & 9,2 \\
\hline $9 . / \mathrm{IV}$. & 1210 & 0,917 & 0,037 & 21,7 & $193, \check{b}$ & 126,9 & 66,6 & 65,6 & 34,4 & 8,9 \\
\hline 10./IV. & 1140 & 0,854 & 0,039 & 20,8 & 171,7 & 115,1 & 56,6 & 67,2 & 32,8 & 8,25 \\
\hline 11./IV. & 1630 & 0,941 & 0,033 & 22,5 & 176,5 & 117,7 & 58,8 & 66,7 & 33,3 & 7,85 \\
\hline & & & & 22,1 & 191,1 & 126,6 & 64,5 & 66,3 & 33,7 & 8,7 \\
\hline
\end{tabular}

N., 38 Jahre alt. 64 Kilo.

\begin{tabular}{|c|c|c|c|c|c|c|c|c|c|c|}
\hline \multirow{3}{*}{ Tag } & \multirow{3}{*}{$\begin{array}{c}\text { Volum } \\
\text { ecm }\end{array} \mid$} & \multicolumn{9}{|c|}{ Mittelzahlen } \\
\hline & & \multirow{2}{*}{$\begin{array}{c}\mathrm{H} \\
\text { (Harn- } \\
\text { säure) }\end{array}$} & \multirow{2}{*}{$\underset{\text { (Xörper) }}{\mathrm{X}}$} & \multirow{2}{*}{$\mathrm{N}$} & \multirow{2}{*}{ NTO' } & \multirow{2}{*}{ UTO' } & \multirow{2}{*}{ RTO' } & \multicolumn{2}{|c|}{$\mathrm{NTO}^{\prime}=100$} & \multirow{2}{*}{$\frac{\text { NTO }}{\mathrm{N}}$} \\
\hline & & & & & & & & UTO' & RTO & \\
\hline 7./IV. & $16 \check{6}$ & 1,012 & 0,082 & 23,1 & 221,1 & 136,6 & 84,5 & 61,8 & 38,2 & 9,6 \\
\hline 8./IV. & 1620 & 0,976 & 0,0 & 22,5 & $20 j, 7$ & 131,2 & 74,5 & 63,7 & 36,3 & 9,1 \\
\hline 9./IV. & 1548 & 0,984 & 0,047 & 21,4 & 212,4 & 143,2 & 69,2 & 67,4 & 32,6 & 9,9 \\
\hline 10./IV. & 1490 & 0,994 & $0,05 \overline{4}$ & 20,9 & 206,0 & 134,0 & 72,0 & 65,0 & 35,0 & 9,85 \\
\hline 11./[V. & 1545 & 0,964 & 0,043 & 21,0 & 197,6 & 130,0 & 67,6 & 65,8 & 34,2 & 9,4 \\
\hline & & & & 21,8 & 208,6 & 135,0 & 73,6 & 64,7 & 35,3 & 9,6 \\
\hline
\end{tabular}

Reihe III. Oxydationszahlen in der Tagesmenge bei gemischter gleichmässiger, in denselben Zeiten genossener Nahrung, bei Stickstoffgleichgewicht und bei gleicher Absonderung des Stickstoffs - der Harnsäure ${ }^{1}$ ) und der Restkörper. - Bei 2 Personen.

1) Siehe M. Krüger. 
G., 2ら Jahre alt.

\begin{tabular}{|c|c|c|c|c|c|c|c|c|c|c|c|c|c|}
\hline \multirow{3}{*}{ Tag } & \multirow{3}{*}{\begin{tabular}{|c|} 
Volum \\
$\mathrm{ecm}$
\end{tabular}} & \multicolumn{12}{|c|}{ Mittelzahlen } \\
\hline & & \multirow{2}{*}{$\begin{array}{c}\text { H } \\
\text { (Harn- } \\
\text { säure) }\end{array}$} & \multirow{2}{*}{$\mid \begin{array}{c}\mathrm{X} \\
\text { (Xanthin- } \\
\text { körper) }\end{array}$} & \multirow{2}{*}{$\mathbf{N}$} & \multirow{2}{*}{ NTO' } & \multirow{2}{*}{ UTO' } & \multirow{2}{*}{ RTO } & \multirow{2}{*}{ NTO } & \multirow{2}{*}{ UTO } & \multirow{2}{*}{ RTO } & \multicolumn{2}{|c|}{$\mathrm{NTO}^{\prime}=100$} & \multirow{2}{*}{$\frac{\mathrm{NTO}^{\prime}}{\mathrm{N}}$} \\
\hline & & & & & & & & & & & UTO & $|\mathrm{RTO}|$ & \\
\hline 7./V. & 1083 & 0,8099 & 0,0244 & 21,81 & 170,73 & 109,43 & 61,30 & 110,42 & $8 \tilde{0}, 25$ & 25,17 & 64,07 & 35,90 & 7,84 \\
\hline 8./V. & 1210 & 0,8020 & 0,0171 & 22,22 & 172,34 & 108,38 & 63,96 & 107,60 & 84,42 & 23,18 & 62,88 & 37,12 & 7,7 \\
\hline 9./V. & 1250 & 0,8157 & 0,0232 & 22,79 & 180,71 & 110,24 & 70,47 & 110,80 & 85,86 & 24,94 & 61,00 & 39,00 & 7,90 \\
\hline & Im & Mittel & . $\cdot$ & 22,30 & 174,59 & 109,34 & 65,24 & 109,61 & $8 \check{0}, 18$ & 24,43 & 62,62 & 37,38 & 7,83 \\
\hline
\end{tabular}

\begin{tabular}{|c|c|c|c|c|c|c|c|c|c|c|c|c|c|}
\hline \multirow{3}{*}{$\mathrm{Tag}$} & \multirow{3}{*}{$\begin{array}{c}\text { Volum } \\
\mathrm{ccm}\end{array}$} & \multicolumn{12}{|c|}{ Mittelzahlen } \\
\hline & & \multirow{2}{*}{\begin{tabular}{|c|}
$\mathrm{H}$ \\
(Harn- \\
säure)
\end{tabular}} & \multirow{2}{*}{$\underset{\substack{\mathrm{X} \\
\text { (Xanthin- } \\
\text { körper) }}}{-}$} & \multirow{2}{*}{$\mathrm{N}$} & \multirow{2}{*}{ NTO' } & \multirow{2}{*}{ UTO' } & \multirow{2}{*}{$\mathrm{RTO}^{\prime}$} & \multirow{2}{*}{ NTO } & \multirow{2}{*}{ UTo. } & \multirow{2}{*}{ RTO } & \multicolumn{2}{|c|}{$\mathrm{NTO}^{\prime}=100$} & \multirow{2}{*}{$\frac{\text { NTO }}{\mathrm{N}}$} \\
\hline & & & & & & & & & & & $\mathrm{UTO}^{\prime}$ & $|\overline{\mathrm{RTO}}|$ & \\
\hline 7./ V. & $149 \check{5}$ & 0,8457 & 0,0223 & 21,96 & $17 \check{\jmath}, 7 \check{\jmath}$ & 114,27 & 61,48 & 114,26 & 89,02 & 25,24 & 64,99 & $3 \tilde{\mathbf{s}}, 01$ & 8,00 \\
\hline 8./V. & 1350 & 0,8511 & 0,0229 & 22,42 & 175,89 & 115,01 & 60,88 & 116,00 & $89, \check{9} 9$ & 26,4 & $6 \check{0}, 38$ & 34,62 & 7,80 \\
\hline 9./V. & 1: $\div 30$ & 0,8461 & 0,0223 & 22,26 & 175,63 & 111,13 & $64, \check{1} 1$ & 116,33 & 89,06 & 27,27 & 67,09 & 32,91 & 7,89 \\
\hline & Im & ittel & & 22,21 & 175,76 & $11:, 40$ & 61,22 & $115, \overline{5}: 3$ & 89,22 & 26,31 & 65,76 & 34,24 & 7,89 \\
\hline
\end{tabular}

Zusammenstellung der Mittel der grössten und kleinsten Zahlen aus den Reihen I-III.

Reihe I.

\begin{tabular}{|c|c|c|c|c|c|c|c|c|c|c|}
\hline \multirow{3}{*}{$\begin{array}{l}\text { Versuchs- } \\
\text { person } \\
\text { und Alter }\end{array}$} & \multicolumn{2}{|c|}{ NTO } & \multicolumn{2}{|c|}{$\mathrm{UTO}^{\circ}$} & \multicolumn{2}{|c|}{$\mathrm{RTO}^{\prime}$} & \multicolumn{4}{|c|}{$\mathrm{NTO}^{\prime}=100$} \\
\hline & \multirow[b]{2}{*}{$\begin{array}{l}\text { Mittel- } \\
\text { werth }\end{array}$} & \multirow{2}{*}{$\begin{array}{c}\text { Grösste } \\
\text { und } \\
\text { vleinste } \\
\text { ZahJ }\end{array}$} & \multirow[b]{2}{*}{$\begin{array}{l}\text { Mittel- } \\
\text { werth }\end{array}$} & \multirow{2}{*}{$\begin{array}{c}\text { Grösste } \\
\text { und } \\
\text { kleinste } \\
\text { zahl }\end{array}$} & \multirow[b]{2}{*}{$\begin{array}{l}\text { Wittel- } \\
\text { werth }\end{array}$} & \multirow{2}{*}{$\begin{array}{c}\text { Grösste } \\
\text { und } \\
\text { vleinste } \\
\text { Zabli } \\
\end{array}$} & \multicolumn{2}{|c|}{ LTO } & \multicolumn{2}{|c|}{$\mathrm{RTO}^{\prime}$} \\
\hline & & & & & & & $\begin{array}{l}\text { Yittel- } \\
\text { werth }\end{array}$ & 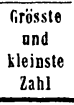 & $\begin{array}{l}\text { Mittel- } \\
\text { merth }\end{array}$ & $\begin{array}{c}\text { Grösste } \\
\text { und } \\
\text { vleinste } \\
\text { Zahl } \\
\end{array}$ \\
\hline $\begin{array}{l}\text { Z. } \\
22 \text { Jahre }\end{array}$ & 163,3 & $\begin{array}{l}181,2 \\
137,2\end{array}$ & 110,9 & $\begin{array}{r}136,4 \\
79,5\end{array}$ & 52,4 & $\begin{array}{l}60,3 \\
48,0\end{array}$ & 67,4 & $\begin{array}{l}72,9 \\
58,8\end{array}$ & 32,6 & $\begin{array}{l}41,2 \\
27,1\end{array}$ \\
\hline $\begin{array}{l}\text { G. } \\
2 j \text { Jahre }\end{array}$ & 151,6 & $\begin{array}{l}178,1 \\
138,4\end{array}$ & $8 \check{5}, \check{\check{c}}$ & $\begin{array}{r}101,0 \\
68,7\end{array}$ & 66,1 & $\begin{array}{l}77,1 \\
55,7\end{array}$ & $\check{5} 7, \check{\jmath}$ & $\begin{array}{l}61,3 \\
\text { อ̆ } \check{5}, 2\end{array}$ & $42, \overline{5}$ & $\begin{array}{l}44,8 \\
38,7\end{array}$ \\
\hline $\begin{array}{l}\mathrm{T} . \\
26 \text { Jahre }\end{array}$ & 1508,7 & $\begin{array}{l}212,7 \\
134,6\end{array}$ & 90,7 & $\begin{array}{r}125,5 \\
68,3\end{array}$ & 68,0 & $\begin{array}{l}87,2 \\
51,4\end{array}$ & $\check{5} 6,9$ & $\begin{array}{l}59,0 \\
\text { วั5,4 }\end{array}$ & 43,1 & $\begin{array}{l}44,6 \\
41,0\end{array}$ \\
\hline $\begin{array}{l}\text { A. } \\
30 \text { Jahre }\end{array}$ & 181,4 & $\begin{array}{l}187,4 \\
171,1\end{array}$ & 99,9 & $\begin{array}{r}104,8 \\
96, \check{5}\end{array}$ & $81, \check{5}$ & $\begin{array}{l}89,3 \\
72,6\end{array}$ & วั5̆,0 & $\begin{array}{l}57,3 \\
51,7\end{array}$ & 45,0 & $\begin{array}{l}48,3 \\
42,7\end{array}$ \\
\hline
\end{tabular}


Die Oxydationszahlen des Harns in saurer u. alkalischer Lösung. 181

Reihe II.

\begin{tabular}{|c|c|c|c|c|c|c|c|c|c|c|c|c|}
\hline \multirow{3}{*}{$\begin{array}{l}\text { Versuchs- } \\
\text { person } \\
\text { und Alter }\end{array}$} & \multicolumn{2}{|c|}{ NTO' } & \multicolumn{2}{|c|}{ UTO } & \multicolumn{2}{|c|}{ RTO } & \multicolumn{4}{|c|}{$\mathrm{NTO}^{\prime}=100$} & \multirow{2}{*}{\multicolumn{2}{|c|}{$\frac{\mathrm{NTO}^{\prime}}{\mathrm{N}}$}} \\
\hline & \multirow[b]{2}{*}{$\begin{array}{c}\text { Mittel- } \\
\text { merth }\end{array}$} & \multirow{2}{*}{$\begin{array}{c}\text { Grösste } \\
\text { und } \\
\text { vleinste } \\
\text { Zabl }\end{array}$} & \multirow[b]{2}{*}{$\begin{array}{c}\text { Mittel- } \\
\text { werth }\end{array}$} & \multirow{2}{*}{$\begin{array}{c}\text { Grösste } \\
\text { und. } \\
\text { Hlejnste } \\
\text { Zahl }\end{array}$} & \multirow[b]{2}{*}{$\begin{array}{l}\text { Wittel- } \\
\text { werth }\end{array}$} & \multirow{2}{*}{$\begin{array}{c}\text { Grösste } \\
\text { und } \\
\text { veinste } \\
\text { Zahl }\end{array}$} & \multicolumn{2}{|c|}{ UTO } & \multicolumn{2}{|c|}{ RTO' } & & \\
\hline & & & & & & & $\begin{array}{l}\text { Mittel- } \\
\text { verth }\end{array}$ & $\begin{array}{c}\text { Grössste } \\
\text { und } \\
\text { vleinste } \\
\text { Zabl }\end{array}$ & $\begin{array}{l}\text { Mittel- } \\
\text { rerth }\end{array}$ & $\begin{array}{c}\text { Grösste } \\
\text { und } \\
\text { vleinste } \\
\text { zahl }\end{array}$ & $\begin{array}{l}\text { Wiltel- } \\
\text { worth }\end{array}$ & $\begin{array}{c}\text { Grössto } \\
\text { uad } \\
\text { vleinste } \\
\text { Zahl }\end{array}$ \\
\hline G. & 1 & 208,9 & & 141,5 & & 72,6 & ? & 67,7 & 27 & 35,5 & & 9,5 \\
\hline 2ら Jahre & 91,1 & 171,7 & $126, b$ & 115,1 & 64,5 & 56,3 & $b, 3$ & 64,5 & 33,7 & 32,8 &, 7 & 7,85 \\
\hline $\mathrm{N}$. & 2086 & 221,1 & 135.0 & 143,2 & 73.6 & 84,5 & 647 & 67,4 & 35.3 & 38,2 & 9.6 & 9,9 \\
\hline 38 Jahre & & 197,6 & 100,0 & 140,0 & & 67,6 & & 61,8 & & 32,6 & & 9,1 \\
\hline
\end{tabular}

Reihe III.

\begin{tabular}{|c|c|c|c|c|c|c|c|c|c|c|c|c|}
\hline \multirow{3}{*}{$\begin{array}{l}\text { Versuchs- } \\
\text { person } \\
\text { und Alter }\end{array}$} & \multicolumn{2}{|c|}{ NTO } & \multicolumn{2}{|c|}{ UTO } & \multicolumn{2}{|c|}{ RTO } & \multicolumn{4}{|c|}{$\mathrm{NTO}^{\prime}=100$} & \multirow{2}{*}{\multicolumn{2}{|c|}{$\frac{\mathrm{NTO}^{\prime}}{\mathrm{N}}$}} \\
\hline & \multirow[b]{2}{*}{$\begin{array}{l}\text { Mittel- } \\
\text { werth }\end{array}$} & \multirow[b]{2}{*}{$\begin{array}{c}\text { Grösste } \\
\text { und } \\
\text { vieínste } \\
\text { Zahl }\end{array}$} & \multirow[b]{2}{*}{$\begin{array}{l}\text { Mittel- } \\
\text { rerth }\end{array}$} & \multirow[b]{2}{*}{$\begin{array}{c}\text { Grösste } \\
\text { und } \\
\text { veinste } \\
\text { Zabl }\end{array}$} & \multirow[b]{2}{*}{$\begin{array}{l}\text { uituel } \\
\text { werth }\end{array}$} & \multirow[b]{2}{*}{$\left|\begin{array}{c}\text { Grösste } \\
\text { und } \\
\text { vleinste } \\
\text { Zahl }\end{array}\right|$} & \multicolumn{2}{|c|}{ UTO } & \multicolumn{2}{|c|}{ RTO } & & \\
\hline & & & & & & & \begin{tabular}{|c|} 
Mittel- \\
verth
\end{tabular} & $\left|\begin{array}{c}\text { Grösste } \\
\text { nnd } \\
\text { kloinste } \\
\text { Zahl }\end{array}\right|$ & \begin{tabular}{|c} 
Mittel- \\
verth
\end{tabular} & \begin{tabular}{|c|} 
Grösste \\
und \\
vleinste \\
Zabl
\end{tabular} \mid & \begin{tabular}{|c|} 
Mittel- \\
werth
\end{tabular} & $\begin{array}{c}\text { Grössto } \\
\text { und } \\
\text { uleinste } \\
\text { Zahl }\end{array}$ \\
\hline 25 Jahre & 174,59 & $\begin{array}{l}180,71 \\
170,73\end{array}$ & 109,34 & $\begin{array}{l}110,24 \\
108,38\end{array}$ & 59 & $\begin{array}{l}70,47 \\
61,30\end{array}$ & 62,6 & $\begin{array}{l}64,07 \\
61,00\end{array}$ & 37,38 & $\begin{array}{l}39,00 \\
35,90\end{array}$ & 7,83 & $\begin{array}{l}7,90 \\
7,75\end{array}$ \\
\hline 38 Jahre & 175,76 & $\begin{array}{l}175,89 \\
175,63\end{array}$ & 113,40 & \begin{tabular}{|l|}
115,01 \\
111,13
\end{tabular} & 61,22 & $\begin{array}{l}61,30 \\
60,54\end{array} \mid$ & 65,76 & $\begin{array}{l}67,09 \\
64,99\end{array}$ & 34,24 & $\left|\begin{array}{l}35,01 \\
32,91\end{array}\right|$ & 7,89 & $\begin{array}{l}8,00 \\
7,80\end{array}$ \\
\hline
\end{tabular}

Aus diesen Tabellen ergibt sich zuerst die interessante Beobachtung, dass das Alter des Individuum auf die Abscheidung der Restkörper von Einfluss ist, indem die durchschnittlichen absoluten Werthe für RTO' mit dem Alter des Individuums steigen.

\begin{tabular}{|c|c|c|c|c|c|}
\hline $\begin{array}{l}\text { Versuchs- } \\
\text { person }\end{array}$ & Alter & RTO & $\begin{array}{l}\text { Anzahl der } \\
\text { Beobachtungs- } \\
\text { tage }\end{array}$ & $\underset{\text { Monate }}{\text { Im }}$ & \\
\hline $\mathrm{Z}$. & 22 Jahre & 52,4 & 7 & Februar & Kost gleichmässig \\
\hline G. & 25 & 66,1 & 6 & $\triangleright$ & gemischt. Körper- \\
\hline$\triangleright$ & 25 & 66,3 & $\check{\jmath}$ & April & grösse, Arbeitszeit \\
\hline » & 25 & $65 \check{5}, 2$ & 3 & Mai & annähernd gleich. \\
\hline $\mathrm{T}$. & 26 & 68,0 & 4 & Februar & \\
\hline A. & 30 & 81,5 & 3 & ১ & \\
\hline N. & 38 & 73,6 & 5 & April & \\
\hline s & 38 & 61,2 & 3 & Mai & \\
\hline
\end{tabular}

Hoppe-Seyler's Zeitschrift f. physiol. Chemie. XXXVI. 
Die Unterschiede sind nur bei grösseren Beobachtungsreihen ausgeprägt. Es können wie bei N. offenbar Perioden vorkommen, wo die Restoxydationszahl durch den Einfluss der Diät, der Beschäftigung, der Jahreszeit und wohl auch andere Einflüsse unter die Altersnormale fällt oder etwas über dieselbe steigt. ${ }^{1}$ )

Mit diesem Vorbehalt kann man aus den angeführten Daten folgende normale Zahlen und deren Grenzen ableiten.

\begin{tabular}{|c|c|c|c|c|c|c|}
\hline \multirow{2}{*}{$\begin{array}{c}\text { Für das Alter } \\
\text { von } 22-38 \text { Jahren }\end{array}$} & \multirow{2}{*}{ NTO' } & \multirow{2}{*}{ UTO' } & \multirow{2}{*}{ RTO' } & \multicolumn{2}{|c|}{$\begin{array}{c}\text { Auf } 100 \text { NTO } \\
\text { entfallen }\end{array}$} & \multirow{2}{*}{$\frac{\mathrm{NTO}^{\prime}}{\mathrm{N}}$} \\
\hline & & & & NTO & RTO & \\
\hline Mittelzahlen . . & 175,6 & 108,9 & 66,5 & 61,5 & $38, \tilde{b}$ & 8,5 \\
\hline Die höchste Zahl . & 221.1 & 143,2 & 89,3 & $\check{51,7}$ & 48,3 & 9,6 \\
\hline Die niedrigste Zahl & 134,6 & 68,3 & 51,4 & 73,0 & 27,0 & $7,8: 3$ \\
\hline $\begin{array}{l}\text { Die am häufigsten } \\
\text { vorkommendeZahl }\end{array}$ & 175,0 & 115,0 & 61,0 & 64,0 & 36,0 & - \\
\hline
\end{tabular}

Man sieht daraus, dass in normalen Verhältnissen RTO' niemals grösser ist als $\mathrm{NTO}^{\prime}$ und dass der kalorische Verlust $\frac{\mathrm{NTO}^{\prime}}{\mathrm{N}}$ niemals die Zahl 10 übersteigt.

Um nun zu prüfen, wie sich die Oxydationszahlen unter dem Einfluss des Verdauungsprocesses ändern, haben wir die Tagesmenge des Harns in zwei Portionen untersucht, und zwar gesondert den Theil, welcher zwischen 12 Uhr Mittags und $12 \mathrm{Uhr}$ Nachts abgeschieden wurde (I. Verdauungsperiode) und gesondert den Harn von 12 Uhr Nachts bis 12 Uhr Mittags (II. Verdauungsperiode).

Die Summen dieser beiden Verdauungsperioden sind bereits in den Reihen II und III angeführt worden.

1) An dieser Stelle muss ich bemerken, dass die Bacterienwirkung auf den Harn möglichst auszuschliessen ist - und dass uns Kinderharne vorgekommen sind, deren RTO-Zahlen dem obigen Altersgesetz nicht entsprachen. 
Die Oxydationszahlen des Harns in saurer u. alkalischer Lösung. 183
G. Reihe II A. I. Verdauungsperiode (12 Uhr Mittags bis 12 Uhr Nachts).

\begin{tabular}{|c|c|c|c|c|c|c|c|c|c|c|}
\hline \multirow{3}{*}{$\mathrm{Tag}$} & \multirow{3}{*}{$\begin{array}{c}\text { Volum } \\
\text { ccm }\end{array}$} & \multicolumn{9}{|c|}{ Mittelzahlen } \\
\hline & & \multirow{2}{*}{$\mathbf{H}$} & \multirow[b]{2}{*}{$\boldsymbol{\Lambda}$} & \multirow{2}{*}{$\mathbf{N}$} & \multirow{2}{*}{ NTO' } & \multirow{2}{*}{ UTO' } & \multirow{2}{*}{ RTO } & \multicolumn{2}{|c|}{$\mathrm{NTO}^{\circ}=100$} & \multirow{2}{*}{$\frac{\text { NTO }^{\prime}}{\mathrm{N}}$} \\
\hline & & & & & & & & UTO' & RTO' & \\
\hline 9./IV. & 800 & 0,642 & 0,023 & 13,1 & 132,8 & 89,6 & 43,2 & 67,5 & 32,5 & 10,1 \\
\hline 10./IV. & 700 & 0,523 & 0,024 & 10,8 & 102,2 & 70,7 & 31,5 & 69,1 & 30,9 & 9,46 \\
\hline 11./IV. & 1050 & 0,615 & 0,021 & 12,9 & 103,4 & 73,0 & 30,4 & 70,6 & 29,4 & 8,01 \\
\hline \multicolumn{2}{|l|}{$\operatorname{Im} 1$} & 0,593 & 0,023 & 12,3 & 112,8 & 77,8 & 35,0 & 69,1 & 30,9 & - \\
\hline
\end{tabular}

G. Reihe II B. II. Verdauungsperiode (12 Uhr Nachts bis 12 Uhr Mittags).

\begin{tabular}{|c|c|c|c|c|c|c|c|c|c|c|}
\hline \multirow{3}{*}{ Tag } & \multirow{3}{*}{$\begin{array}{c}\text { Volum } \\
\text { ccm }\end{array}$} & \multicolumn{9}{|c|}{ Mittelzahlen } \\
\hline & & \multirow{2}{*}{ H } & \multirow{2}{*}{$\mathbf{X}$} & \multirow{2}{*}{$\mathbf{N}$} & \multirow{2}{*}{ NTO } & \multirow{2}{*}{ UTO' } & \multirow{2}{*}{ RTO' } & \multicolumn{2}{|c|}{$\mathrm{NTO}^{\prime}=100$} & \multirow{2}{*}{$\frac{\text { NTO }^{\prime}}{\mathrm{N}}$} \\
\hline & & & & & & & & UTO & $\overline{\text { RTO }}$ & \\
\hline 9./IV. & 410 & 0,275 & 0,014 & 8,5 & 60.7 & 37,3 & 23,4 & 61,4 & 38,6 & 7,14 \\
\hline 10./IV. & 440 & 0,331 & 0,015 & 10,0 & 69,5 & 44,4 & 25,1 & 63,9 & 36,1 & 6,95 \\
\hline 11./IV. & 580 & 0,329 & 0,012 & 9,6 & 73,1 & 44,7 & 28,4 & 61,1 & 38,9 & 7,6 \\
\hline \multicolumn{2}{|c|}{ Im Mit } & 0,312 & 0,014 & 9,4 & 67,8 & 42,1 & 25,6 & 62,1 & 37,9 & - \\
\hline
\end{tabular}

N. Reihe II A. I. Verdauungsperiode.

\begin{tabular}{|c|c|c|c|c|c|c|c|c|c|c|}
\hline \multirow{3}{*}{ Tag } & \multirow{3}{*}{$\begin{array}{l}\text { Volum } \\
\text { ccm }\end{array}$} & \multicolumn{9}{|c|}{ Mittelzahlen } \\
\hline & & \multirow{2}{*}{$\mathrm{H}$} & \multirow[b]{2}{*}{$X$} & \multirow{2}{*}{$\mathbf{N}$} & \multirow{2}{*}{ NTO } & \multirow{2}{*}{ UTO } & \multirow{2}{*}{ RTO } & \multicolumn{2}{|c|}{$\mathrm{NTO}^{\prime}=100$} & \multirow{2}{*}{$\frac{\text { NTO }^{\prime}}{\mathbf{N}}$} \\
\hline & & & & & & & & UTO & RTO & \\
\hline 9./IV. & 950 & 0,582 & 0,029 & 11,8 & 126,3 & 88,8 & 37,5 & 70,3 & 29,7 & 10,7 \\
\hline 10./IV. & 900 & 0,588 & 0,032 & 11,4 & 115,2 & 79,2 & 36,0 & 68,8 & 31,2 & 10,1 \\
\hline 11./IV. & 800 & 0,445 & 0,018 & 8,96 & 89,6 & 60,0 & 29,6 & 66,9 & 33,1 & 10,0 \\
\hline \multicolumn{2}{|c|}{ Im Mittel } & 0,538 & 0,026 & 10,7 & 110,4 & 76,0 & 34,7 & 68,7 & 31,3 & - \\
\hline
\end{tabular}


N. Reihe II B. II. Verdauungsperiode.

\begin{tabular}{|c|c|c|c|c|c|c|c|c|c|c|}
\hline \multirow{3}{*}{ Tag } & \multirow{3}{*}{$\begin{array}{c}\text { Volum } \\
\mathrm{ccm}\end{array}$} & \multicolumn{9}{|c|}{ Mittelzahlen } \\
\hline & & \multirow{2}{*}{ H } & \multirow{2}{*}{$\mathrm{x}$} & \multirow{2}{*}{$\mathbf{N}$} & \multirow{2}{*}{ NTO } & \multirow{2}{*}{ UTO' } & \multirow{2}{*}{ RTO } & \multicolumn{2}{|c|}{ NTO $=100$} & \multirow{2}{*}{$\frac{\text { NTO }}{\mathrm{N}}$} \\
\hline & & & & & & & & UTO & RTO $^{\prime}$ & \\
\hline 9./IV. & 598 & 0,402 & 0,018 & 9.6 & 86,1 & 54,4 & 31,7 & 63,2 & 36,8 & 8,97 \\
\hline 10./IV. & 590 & 0,406 & 0,022 & 9,5 & 90,8 & 54,8 & 36,0 & 60,3 & 39,7 & $9,5 \check{~}$ \\
\hline 11./IV. & 745 & 0,519 & 0,025 & 12,0 & 108,0 & 70,0 & 38,0 & 64,8 & $3 \check{5}, 2$ & 9,00 \\
\hline & 0,442 & 0,022 & 10,4 & 94,9 & $\check{\jmath} 9,7$ & 35,2 & 62,8 & 37,2 & - \\
\hline
\end{tabular}

Dieselben Verhältnisse bei Stickstoffgleichgewicht und gleicher Abscheidung von Stickstoffharnsäure und Restkörpern:

G. Reihe III A. I. Verdauungsperiode.

\begin{tabular}{|c|c|c|c|c|c|c|c|c|c|c|c|c|c|}
\hline \multirow{3}{*}{ Tag } & \multirow{3}{*}{$\begin{array}{l}\text { Volum } \\
\text { ccm }\end{array}$} & \multicolumn{12}{|c|}{ Mittelzahlen } \\
\hline & & \multirow{2}{*}{ I } & & \multirow[b]{2}{*}{ I } & \multirow{2}{*}{ NTO' } & \multirow{2}{*}{ UT0' } & \multirow{2}{*}{ RTO } & \multirow{2}{*}{ NTO } & \multirow{2}{*}{ UTO } & \multirow{2}{*}{ RTO } & \multicolumn{2}{|c|}{$\mathrm{NTO}^{\prime}=100$} & \multirow{2}{*}{$\frac{\text { NTO }}{\mathrm{N}}$} \\
\hline & & & & & & & & & & & UTO & RTO & \\
\hline . $\mathrm{V}$. & 700 & $0, \overline{5} 511$ & 0,0161 & 12,91 & 111,00 & 74,40 & 36,60 & 74,37 & 58,01 & 16,36 & 67,0 & 33,0 & 8,6 \\
\hline $3 . / \mathrm{V}$. & 820 & 0,5596 & 0,0093 & 13,29 & 113,65 & 75,62 & 38,03 & 74,62 & 58,91 & 15,72 & 66,5 & $33, \tilde{5}$ & 8,5 \\
\hline N. & 760 & 0,5235 & 0,0130 & 12,46 & 108,68 & 70,76 & $|37,92|$ & 69,54 & 55,11 & 14,43 & 65,1 & 34,9 & 8,7 \\
\hline \multicolumn{2}{|c|}{ Im Mit } & - & - & 12,89 & 111,11 & 73,59 & $|37,52|$ & 72,84 & 57,34 & 15,50 & 66,1 & 33,9 & 8,6 \\
\hline
\end{tabular}

G. Reihe III B. II. Verdauungsperiode.

\begin{tabular}{|c|c|c|c|c|c|c|c|c|c|c|c|c|c|}
\hline \multirow{3}{*}{ Tag } & \multirow{3}{*}{\begin{tabular}{|l} 
Volum \\
ccm
\end{tabular}} & \multicolumn{12}{|c|}{ Mittelzahlen } \\
\hline & & \multirow{2}{*}{ H } & \multirow{2}{*}{$\mathrm{x}$} & \multirow{2}{*}{$\mathrm{N}$} & \multirow{2}{*}{ NTO' } & \multirow{2}{*}{ UT0' } & \multirow{2}{*}{ RTO } & \multirow{2}{*}{ NTo } & \multirow{2}{*}{ UTO } & \multirow{2}{*}{ RTO } & \multicolumn{2}{|c|}{$\mathrm{NTO}^{\prime}=100$} & \multirow{2}{*}{$\frac{\text { NTO }}{\mathrm{N}}$} \\
\hline & & & & & & & & & & & UTO' & RTO & \\
\hline 7./V. & 383 & 0,2588 & 0,0083 & 8,90 & 59,73 & 35,00 & 24,73 & 36,05 & 27,24 & 8,81 & 70,6 & 29,4 & 6,71 \\
\hline $8 . / \mathrm{V}$. & 390 & 0,2424 & 0,0078 & 8,94 & 58,69 & 32,76 & 25,93 & 33,98 & 25,51 & 8,47 & 79,1 & 20,9 & 6,5̆5 \\
\hline 9./V. & 490 & 0,2922 & 0,0102 & 10,33 & 72,03 & 39,48 & 32,55 & 41,26 & 30,75 & 10,51 & 82,4 & 17,6 & 6,9 \\
\hline \multicolumn{2}{|c|}{ Im Mit } & - & - & 9,39 & 63,48 & 35,75 & 27,73 & 37,09 & 27,83 & 9,2 & 77,9 & 22,1 & 6,7 \\
\hline
\end{tabular}


Die Oxydationszahlen des Harns in saurer u. alkalischer Lösung. 185

N. Reihe III A. I. Verdauungsperiode.

\begin{tabular}{|c|c|c|c|c|c|c|c|c|c|c|c|c|c|}
\hline \multirow{3}{*}{ Tag } & \multirow{3}{*}{$\begin{array}{c}\text { Volum } \\
\mathrm{ccm}\end{array}$} & \multicolumn{12}{|c|}{ Mittelzahlen } \\
\hline & & \multirow{2}{*}{$\mathbf{H}$} & \multirow{2}{*}{$\mathrm{x}$} & \multirow{2}{*}{$\mathrm{N}$} & \multirow{2}{*}{ NTO' } & \multirow{2}{*}{ UTO' } & \multirow{2}{*}{ RTO' } & \multirow{2}{*}{ NTO } & \multirow{2}{*}{ UTO' } & \multirow{2}{*}{ RTO } & \multicolumn{2}{|c|}{$\mathrm{NTO}^{\prime}=100$} & \multirow{2}{*}{$\frac{\mathrm{NTO}^{\prime}}{\mathrm{N}}$} \\
\hline & & & & & & & & & & & UTO & $\mid \mathrm{RTO}^{\prime}$ & \\
\hline 7./V. & 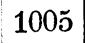 & 0,5226 & 0,0139 & 13,01 & 114,40 & 70,62 & 43,78 & 71,36 & 55,01 & 16,35 & 61,73 & 38,27 & 8,8 \\
\hline 8./V. & 790 & 0,4960 & 0,0112 & 12,36 & 102,70 & 67,03 & 35,67 & 68,34 & 5ூ2,22 & 16,12 & 65,26 & 34,74 & 8,3 \\
\hline 9./V. & 840 & 0,4868 & 0,0111 & 11,97 & 97,69 & 62,58 & 35,11 & 65,53 & 51,24 & 14,29 & 64,05 & 35,95 & 8,2 \\
\hline & Im & 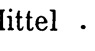 & 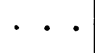 & 12,45 & 104,93 & 66,74 & 38,19 & 68,41 & $\tilde{5} 2,82$ & 15,59 & 63,6 & 36,40 & 8,4 \\
\hline
\end{tabular}

N. Reihe III B. II. Verdauungsperiode.

\begin{tabular}{|c|c|c|c|c|c|c|c|c|c|c|c|c|c|}
\hline \multirow{3}{*}{$\mathrm{Tag}$} & \multirow{3}{*}{$\begin{array}{l}\text { Volum } \\
\mathrm{ccm}\end{array}$} & \multicolumn{12}{|c|}{ Mittelzahlen } \\
\hline & & \multirow{2}{*}{$\mathbf{H}$} & \multirow{2}{*}{$\mathbf{x}$} & \multirow{2}{*}{$\mathbf{N}$} & \multirow{2}{*}{ NTO } & \multirow{2}{*}{ UTO } & \multirow{2}{*}{ RTO' } & \multirow{2}{*}{ NTO } & \multirow{2}{*}{ UTO } & \multirow{2}{*}{ RTO: } & \multicolumn{2}{|c|}{$\mathrm{NTO}^{\prime}=100$} & \multirow{2}{*}{$\frac{\mathrm{NTO}^{\prime}}{\mathrm{N}}$} \\
\hline & & & & & & & & & & & UTO & RTO & \\
\hline 7./V. & 490 & 0,3231 & 0,0084 & 8,95 & 61,35 & 43,65 & 17,70 & 42,90 & 34,01 & 8,89 & 71,1 & 28,9 & 6,85 \\
\hline $8 . / \mathrm{V}$. & 560 & 0,3551 & 0,0117 & 10,06 & 73,19 & 47,98 & 25,21 & 47,66 & 37,37 & 10,29 & 65,5 & 34,5 & 7,27 \\
\hline 9./V. & 590 & 0,3593 & 0,0112 & 10,29 & 77,94 & $48, \tilde{5} 5$ & 29,39 & 50,80 & 37,82 & 12,78 & 62,2 & 37,8 & 7,57 \\
\hline & Im & & & 9,77 & 70,83 & 46,73 & 24,10 & 47,12 & 36,40 & 10,72 & 65,9 & 34,1 & 7,2 \\
\hline
\end{tabular}

Aus diesen Beobachtungen ergibt sich:

1. In der I. Verdauungsperiode sind sowohl die Zahlen für $\mathrm{N}$, als auch diejenigen für $\mathrm{NTO}^{\prime}$, UTO' und RTO' grösser als in der II. Periode.

2. Der Unterschied ist in den Oxydationswerthen viel schärfer ausgeprägt, als in den $\mathrm{N}$-Werthen.

3. Der Unterschied betrifft hauptsächlich die Harnsäureoxydationszahlen UTO‘, während die Restkörperoxydationszahlen weniger differiren.

4. Die Grösse der Differenz zwischen den Zahlen der I. und II. Verdauungsperiode ist bei derselben Ernährung und bei denselben Speiseresten ein Kriterium der Geschwindigkeit, mit welcher der Organismus die Verdauung erledigt. 
G., 25 Jahre alt, weist diesbezüglich folgende Zahlen auf (im Mittel):

\begin{tabular}{|c|c|c|c|c|c|}
\hline & Verdauungsperioden & $\mathrm{N}$ & $\mathrm{NTO}^{\prime}$ & $\mathrm{UTO}^{\prime}$ & $\mathrm{RTO}^{\prime}$ \\
\hline April & I. Periode . . . . & 12,3 & 112,8 & 77,8 & 35,3 \\
\hline \multirow[t]{2}{*}{ » } & II. Periode . . . . & 9,4 & 67,8 & 42,1 & 25,6 \\
\hline & Differenz . . . & 2,9 & 45,0 & 35,9 & 9,7 \\
\hline
\end{tabular}

\begin{tabular}{r|r|r|r|r|r}
\hline \hline & Verdauungsperioden & $\mathrm{N}$ & $\mathrm{NTO}^{\prime}$ & $\mathrm{UTO}^{\prime}$ & $\mathrm{RTO}^{\prime}$ \\
\hline \multirow{4}{*}{} & I. Periode . . . . & 10,7 & 110,4 & 76,0 & 34,7 \\
& II. Periode . . . & 10,4 & 94,9 & 59,7 & 35,2 \\
\hline & Differenz . . . & 0,3 & 15,5 & 16,3 & $-0,5$
\end{tabular}

Die Unterschiede sind sehr ausgesprochen und der Wertl $-0,5$ bedeutet bei $\mathrm{N}$ bereits eine Insufficienz des Stoffumsatzes

Im Mai ändern sich diese Verhältnisse, hier findet mar in den beiden Verdauungsperioden folgende Verhältnisse:

\begin{tabular}{|c|c|c|c|c|c|c|}
\hline & & $\begin{array}{l}\text { Verdauungs- } \\
\text { perioden }\end{array}$ & $\mathrm{N}$ & $\mathrm{NTO}^{\prime}$ & $\mathrm{UTO}^{\prime}$ & $\mathrm{RTO}^{\prime}$ \\
\hline \multirow{3}{*}{ Mai } & & I. Periode . . & 12,89 & 111,11 & 73,59 & 37,32 \\
\hline & & II. Periode . . & 9,39 & 63,48 & 35,75 & 27,77 \\
\hline & G. & Differenz . & 3,50 & 47,63 & 37,84 & 9,55 \\
\hline \multirow{3}{*}{$\begin{array}{c}\text { Mai } \\
»\end{array}$} & & I. Periode . . & 12,45 & 104,93 & 66,74 & 36,67 \\
\hline & & II. Periode . - & 9,77 & 70,83 & 46,73 & 24,10 \\
\hline & N. & Differenz . & 2,68 & 34,1 & 20,01 & 12,57 \\
\hline
\end{tabular}

Man sieht, dass auch hier bei $\mathrm{N}$ die Differenzen geringe: sind, als bei $G$, sie sind hier aber weit grösser als in de Reihe II und ein negativer Werth kommt nicht vor.

Auf diesen verbesserten Stoffumsatz lässt sich wohl auc] 
die Verminderung der RTO-Zahlen zurückführen. Betrachtet man das procentuelle Verhältniss zwischen den Harnsäure-(UT0') und Restkörper- (RTO') Oxydationszahlen in der ersten und in der zweiten Verdauungsperiode, so findet man hier einen Unterschied zwischen April und Mai. Im April findet man in der II. Periode mehr RTO $\mathrm{R}^{\prime} / 0$ als in der ersten. Im Mai ist das Verhältniss umgekehrt.

G.

\begin{tabular}{|c|c|c|c|c|}
\hline \multirow{3}{*}{$\begin{array}{c}\text { Verdauungs- } \\
\text { perioden }\end{array}$} & \multicolumn{2}{|c|}{ April } & \multicolumn{2}{|c|}{ Mai } \\
\hline & \multicolumn{2}{|c|}{$100=$ NTO $^{\prime}$} & \multicolumn{2}{|c|}{$100=\mathrm{NTO}^{\prime}$} \\
\hline & UTO' & $\mathrm{RTO}^{\prime}$ & UTO & RTO $^{\prime}$ \\
\hline I. Periode & 69,1 & 30,9 & 66,1 & 33,9 \\
\hline III. Periode & 62,1 & 37,9 & 76,0 & 24,0 \\
\hline Differ & +7 & $-7,0$ & $-9,9$ & $+9,9$ \\
\hline
\end{tabular}

N.

\begin{tabular}{|c|c|c|c|c|}
\hline \multirow{3}{*}{$\begin{array}{l}\text { Verdauungs- } \\
\text { perioden }\end{array}$} & \multicolumn{2}{|c|}{ April } & \multicolumn{2}{|c|}{ Mai } \\
\hline & \multicolumn{2}{|c|}{$100=\mathrm{NTO}^{\prime}$} & \multicolumn{2}{|c|}{$100=\mathrm{NTO}^{\prime}$} \\
\hline & UTO & RTO' & UTO & $\mathrm{RTO}^{\prime}$ \\
\hline I. Periode . & 68,7 & 31,3 & 63,6 & 36,4 \\
\hline Il. Periode . & 62,8 & 37,2 & 65,9 & 34,1 \\
\hline Differenz . & 150 & 50 & $-2,3$ & $-2,3$ \\
\hline
\end{tabular}

Untersucht man die Ursache dieses Unterschiedes, nämlich, ob die Vermehrung der RTO'Zahl oder die Verminderung der UT0'-Zahl daran Schuld ist, so findet man folgendes:

Bei G.

\begin{tabular}{c|c|c|c}
\hline $\begin{array}{c}\text { Verdauungs- } \\
\text { perioden }\end{array}$ & Monat & UTO $^{\prime}$ & RT0' \\
\hline I. Periode . & April & $\begin{array}{r}77,8 \\
\text { Mai }\end{array}$ & $\begin{array}{r}73,6 \\
35,3 \\
37,3\end{array}$ \\
\hline Differenz . . . . & $+4,2$ & $-2,0$ \\
\hline II. Periode . & April & 42,1 & 25,6 \\
& Mai & 35,8 & 27,8 \\
\hline Differenz . . . . & $+6,3$ & $-2,2$
\end{tabular}

Bei N.

\begin{tabular}{|c|c|c|c|}
\hline $\begin{array}{l}\text { Verdauungs- } \\
\text { perioden }\end{array}$ & Monat & UTO $^{\prime}$ & $\mathrm{RTO}^{\prime}$ \\
\hline I. Periode . & $\begin{array}{l}\text { April } \\
\text { Mai }\end{array}$ & $\begin{array}{l}76,0 \\
66,7\end{array}$ & $\begin{array}{l}34,7 \\
37,1\end{array}$ \\
\hline Differenz . & $\cdot \cdot \cdot$ & $+9,3$ & $-2,4$ \\
\hline II. Periode . & $\begin{array}{l}\text { April } \\
\text { Mai }\end{array}$ & $\begin{array}{l}59,7 \\
46,7\end{array}$ & $\begin{array}{l}35,2 \\
24,1\end{array}$ \\
\hline Diffe & & $+13,0$ & +11 \\
\hline
\end{tabular}

Man sieht, dass im Mai die RT0'-Zahlen nur unbedeutend vermehrt, einmal sogar vermindert sind, während die UTO'Zahlen starke Verminderung im Mai aufweisen. Die Ursache dieser Differenz in der Harnsäureabscheidung ist darauf zurückzuführen, dass im April fetter Lendenbraten, während im Mai 
mageres kaltes Kalbfleisch und Schinken genossen und der Bedarf an Fett durch Butter gedeckt wurde.

Die alkalischen RTO-Zahlen zeigen folgende Verhältnisse: Absolut wird in der I. Verdauungsperiode mehr davon abgeschieden, als in der II. Periode. Relativ zur Harnsäure finden wir bei $G$ und $N$ verschiedene Verhältnisse (siehe 187). Bei $G$ wird in der I. Verdauungsperiode im Vergleiche mit Harnsäure weniger RTO' abgeschieden - bei N beinahe gleichviel. Das hängt damit $a b$, dass $G$ in der I. Verdauungsperiode viel mehr Harnsäure erzeugt als in der Nachperiode, während bei $\mathrm{N}$ in dieser Beziehung der Stoffwechsel träger ist.

Aus den angegebenen Untersuchungen ergeben sich folgende Durchschnittszahlen für die I. und II. Verdauungsperiode bei $\mathrm{G}$ und $\mathrm{N}$.

\begin{tabular}{r|r|r|r|r|r|r|r|r}
\hline \hline Verdauungsperioden & $\mathrm{N}$ & $\mathrm{NTO}^{\prime}$ & $\mathrm{UTO}^{\prime}$ & $\mathrm{RTO}^{\prime}$ & \multicolumn{2}{|c|}{$\mathrm{NTO}^{\prime}=100$} & $\mathrm{NT0}$ \\
\hline I. Periode . . . . & 12,1 & 109,8 & 73,5 & 36,0 & 67,0 & 33,0 & 9,1 \\
II. Periode . . . . . & 9,7 & 75,2 & 46,1 & 28,2 & 61,3 & 38,7 & 7,7 \\
\hline Differenz . . . & 2,4 & 35,2 & 27,4 & 7,8 & - & - & 1,4
\end{tabular}

Die Schwankungen sind hier sehr bedeutend. Sie hängen sowohl vom Alter, als auch von dem Zustande des Darms der Qualität und Quantität der Nahrung und wohl von vielen anderen Umständen $\mathrm{ab}$. Die ermittelten Zahlen geben aber bei bestimmten Esszeiten immer ein werthvolles Mittel zur Beurtheilung des Stoffumsatzes.

Der Einfluss der Kostünderung auf die Oxydationszahlen des Harns.

In weiterer Fortsetzung der Versuche Reihe III hat G. die Diät insofern verändert, als er die Hälfte seiner bisherigen vegetabilischen Nahrung mit stickstoffgleicher und äquikalorischer Menge der animalischen Nahrung vertauschte, und nach Fortsetzung des Versuches durch drei Tage auch den Rest der 
vegetabilischen Nahrung in animalischer Gleichwerthigkeit einnahm. Nach 2 Tagen zeigten sich dann die ersten Anzeichen einer Verdauungsstörung. In Folge dessen wurde am 6. Tage in der Tagesperiode gar keine Nahrung eingenommen und erst 12 Uhr Nachts 2 Eier und 1 Semmel genossen.

$\mathrm{N}$ verwandelte umgekehrt die animalische Nahrung in zwei Etapen in rein vegetabilische (theoretisch äequivalente) Kost und führte den Versuch durch zweimal 3 Tage ununterbrochen durch.

Die Resultate, welche sich dabei ergeben haben, führen wir in den nachstehenden Tabellen an.

Die Verhältnisse lassen sich besser an den beigegebenen graphischen Aufzeichnungen studiren.

Wir heben aber ausdrücklich hervor, dass es uns bei diesen Versuchen nicht auf das Studium der Verhältnisse bei rein animalischer oder rein vegetabilischer Nahrung angekommen ist, denn die Zeitdauer der Versuche wäre für die Feststellung der Stoffwechselnormalzahlen zu gering - wir wollten an diesen Versuchen nur studiren, inwieweit sich bei grösseren Schwankungen die einzelnen Werthe gegenseitig beeinflussen.

G. Oxydationszahlen in der Tagesmenge des Harns.

\begin{tabular}{|c|c|c|c|c|c|c|c|c|c|c|c|c|c|}
\hline \multirow{3}{*}{ Tag } & \multirow{3}{*}{$\begin{array}{l}\text { Volum } \\
\mathrm{crm}\end{array}$} & \multicolumn{12}{|c|}{ Mittelzahlen } \\
\hline & & \multirow{2}{*}{$\mathrm{H}$} & \multirow{2}{*}{$\mathrm{x}$} & \multirow{2}{*}{$\mathbf{N}$} & \multirow{2}{*}{ NTO' } & \multirow{2}{*}{ UTO' } & \multirow{2}{*}{ RTO' } & \multirow{2}{*}{ NTO } & \multirow{2}{*}{ UTO } & \multirow{2}{*}{ RTO. } & \multicolumn{2}{|c|}{$\mathrm{NTO}^{\prime}=100$} & \multirow{2}{*}{$\frac{\text { NTO }}{\mathrm{N}}$} \\
\hline & & & & & & & & & & & UTO & RTO & \\
\hline $.0 . / \mathrm{T}$. & 940 & 0,6500 & 0,0196 & 20,24 & 150,21 & 87,84 & 62,37 & 91,34 & 68,41 & 22,93 & 58,41 & 41,59 & 7,42 \\
\hline $.1 . / \pi$. & 1235 & 0,7215 & 0,0200 & 22,75 & 150,57 & 97,49 & 53,08 & 98,69 & 7 & 22,77 & 62,03 & 37,97 & 6,62 \\
\hline $.2 . / \mathrm{T}$. & 1290 & 0,8501 & 0,0282 & 23,72 & 172,24 & 114,90 & 57,34 & 111,99 & 9,48 & 22,51 & 66,70 & 33,30 & 7,26 \\
\hline $.3 . / \mathrm{T}$. & 1220 & 0,7158 & 0,0265 & 22,69 & 153,35 & 96,73 & 56,62 & 95,27 & 75,3 & 19,9 & 63,08 & 36,92 & 6,76 \\
\hline 4.N. & 1535 & 0,8212 & 0,0310 & 24,52 & 169,85 & 110,97 & 58,88 & 107,96 & 86,43 & 21,53 & 65,33 & 34,67 & 6,92 \\
\hline $.5 . / T$. & 558 & 0,4505 & 0,0256 & 12,50 & 95,11 & 60,74 & 34,37 & 58,36 & 47,42 & 10,94 & 63,86 & 36,14 & 7,60 \\
\hline
\end{tabular}


Oxydationszahlen in der I. Verdauungsperiode.

\begin{tabular}{|c|c|c|c|c|c|c|c|c|c|c|c|c|c|}
\hline \multirow{3}{*}{ Tag } & \multirow{3}{*}{$\begin{array}{l}\text { Dolum } \\
\mathrm{ccm}\end{array}$} & \multicolumn{12}{|c|}{ Mittelzahlen } \\
\hline & & \multirow{2}{*}{$\mathbf{H}$} & \multirow{2}{*}{$X$} & \multirow{2}{*}{$\mathbf{N}$} & \multirow{2}{*}{ NTO } & \multirow{2}{*}{ UTO } & \multirow{2}{*}{ RTO } & \multirow{2}{*}{ NTO } & \multirow{2}{*}{ UTO } & \multirow{2}{*}{ RTO. } & \multicolumn{2}{|c|}{$\mathrm{NTO}^{\prime}=100$} & \multirow{2}{*}{$\frac{\text { NTO }}{\mathrm{N}}$} \\
\hline & & & & & & & & & & & UTO' & $\left|\mathrm{RTO}^{\prime}\right|$ & \\
\hline 10. N. & 560 & 0,4186 & 0,0117 & 10,84 & 89,26 & 56,57 & 32,69 & 58,74 & 44,06 & 14,68 & 63,26 & 36,74 & 8,23 \\
\hline $11 ., \mathrm{N}$. & 895 & 0,5187 & 0,0127 & 14,80 & 104,09 & 70,09 & 34,00 & 69,81 & 54,59 & 15,22 & 67,33 & 32,67 & 7,03 \\
\hline $12 ., \mathrm{V}$. & 740 & $0, \check{1} 128$ & 0,0126 & 12,86 & 101,45 & 69,32 & 32,13 & 66,23 & 53,98 & 12,25 & 68,33 & 31,67 & 7,90 \\
\hline 13./V. & 660 & 0,4242 & 0,0138 & $11, \tilde{2} 2$ & 86,26 & 57,32 & 28,94 & 53,72 & 44,65 & 9,07 & 64,45 & 33,55 & 7,48 \\
\hline $14 . / \mathrm{V}$. & 980 & 0,4774 & 0,0205 & 13,45 & 98,98 & 64,51 & 34,47 & 63,50 & 50,25 & 13,25 & 65,18 & 34,82 & 7,36 \\
\hline $15 . / Y$. & 265 & 0,1847 & 0,0100 & 5,16 & 38,56 & 24,96 & 13,60 & $23,0 \check{5}$ & 19,44 & 3,61 & 64,98 & 35,02 & 7,47 \\
\hline
\end{tabular}

Oxydationszahlen in der II. Verdauungsperiode.

\begin{tabular}{|c|c|c|c|c|c|c|c|c|c|c|c|c|c|}
\hline \multirow{3}{*}{ Tag } & \multirow{3}{*}{$\begin{array}{l}\text { Volum } \\
\text { ccm }\end{array}$} & \multicolumn{12}{|c|}{ Mittelzahlen } \\
\hline & & \multirow{2}{*}{ H } & \multirow{2}{*}{$\mathrm{x}$} & \multirow{2}{*}{$\mathbf{N}$} & \multirow{2}{*}{ NTO' } & \multirow{2}{*}{ UTO' } & \multirow{2}{*}{ RTO } & \multirow{2}{*}{ NTO } & \multirow{2}{*}{ UTO } & \multirow{2}{*}{ RTO } & \multicolumn{2}{|c|}{$\mathrm{NTO}^{\prime}=100$} & \multirow{2}{*}{$\frac{\text { NTO }^{\prime}}{\mathrm{N}}$} \\
\hline & & & & & & & & & & & UTO' & RTO & \\
\hline $10 . \mathrm{V}$. & 380 & 0,2314 & 0,0079 & 9,40 & 60,9อ̃ & 31,27 & 29,68 & 32,60 & 24,35 & $8,2 \tilde{J}$ & 51,30 & 48,70 & 6,18 \\
\hline $11 . / \mathrm{V}$. & 350 & 0,2028 & 0,0073 & 7,95 & 46,48 & 27,40 & 19,08 & 28,88 & 21,33 & $7,5 \check{~}$ & 58,95 & 41,05 & 5,80 \\
\hline $12 . \mathrm{N}$. & 550 & 0,3373 & 0,0156 & 10,86 & 70,79 & 45,58 & 25,21 & 45,76 & 35,50 & 10,26 & 64,30 & $3 \check{,}, 70$ & 6,כ̆1 \\
\hline $13 . ; \mathrm{V}$. & 560 & 0,2916 & 0,0127 & 11,17 & 67,09 & 39,41 & 27,68 & $41, \check{5}$ & 30,69 & 10,86 & $\overline{\mathrm{j}} 8,73$ & 41,27 & 6,00 \\
\hline $14 . / v^{\prime}$ & 555 & 0,3438 & 0,0105 & 11,07 & 70,87 & 46,46 & 24,41 & 44,46 & 36,18 & 8,28 & $65, \check{5} 8$ & 34,42 & 6,40 \\
\hline $1 \check{0} . / \mathrm{V}$. & 293 & 0,2658 & 0,0156 & 7,34 & 56,55 & 35,78 & 20,77 & 35,31 & 27,98 & 7,33 & 63,27 & 36,73 & 7,70 \\
\hline
\end{tabular}

N. Oxydationszahlen in der Tagesmenge des Harns.

\begin{tabular}{|c|c|c|c|c|c|c|c|c|c|c|c|c|c|}
\hline \multirow{3}{*}{ Tag } & \multirow{3}{*}{$\begin{array}{l}\text { Volum } \\
\text { ccm }\end{array}$} & \multicolumn{12}{|c|}{ Mittelzahlen } \\
\hline & & \multirow{2}{*}{$\mathbf{H}$} & \multirow{2}{*}{$\mathrm{x}$} & \multirow{2}{*}{$\mathbf{N}$} & \multirow{2}{*}{ NTO } & \multirow{2}{*}{ UTO' } & \multirow{2}{*}{ RTO } & \multirow{2}{*}{ NTO } & \multirow{2}{*}{ UTO } & \multirow{2}{*}{ RTO. } & \multicolumn{2}{|c|}{$\mathrm{NTO}^{\prime}=100$} & \multirow{2}{*}{$\frac{\mathrm{NTO}}{\mathrm{N}}$} \\
\hline & & & & & & & & & & & UTO' & RTO & \\
\hline $10, \mathrm{~V}$. & 1510 & 0,8433 & 0,0264 & 21,04 & 188,94 & 113,96 & 74.98 & 127,83 & 88,76 & 39,07 & 60,31 & 39,69 & 8,99 \\
\hline $11 . N$. & 1360 & 0,7391 & 0,0210 & 19,47 & 177,38 & 99,88 & 77,50 & 109,00 & 77,79 & 31,21 & 56,23 & 43,77 & 9,11 \\
\hline 12.17. & 1375 & 0,8538 & 0,0316 & 20,73 & 175,37 & 115,37 & 60,00 & 121,71 & 89,88 & 31,83 & 65,78 & 34,22 & 8,46 \\
\hline 13. $\mathrm{N}$. & 1410 & 0,9123 & 0,0277 & 17,28 & $195,3 \dot{0}$ & $12: 3,16$ & 72,19 & 125,66 & 96,03 & 29,6 & 62,02 & 37,98 & 11,30 \\
\hline 14.7. & 1323 & 0,8027 & 0,0343 & 19,93 & 187,17 & 108,47 & 78,70 & 114,99 & 84,49 & 30,50 & 58,00 & 42,00 & 9,40 \\
\hline 15./N. & 1400 & 0,8576 & 0,0454 & 18,71 & $18: 3,45$ & 115,89 & $67, \tilde{5} 6$ & $121,5 \check{5}$ & 90,28 & 31,27 & 63,49 & 37,38 & 9,80 \\
\hline
\end{tabular}


Oxydationszahlen in der I. Verdauungsperiode.

\begin{tabular}{|c|c|c|c|c|c|c|c|c|c|c|c|c|c|}
\hline \multirow{3}{*}{ Tag } & \multirow{3}{*}{$\begin{array}{c}\text { Volum } \\
\mathrm{ccm}\end{array}$} & \multicolumn{12}{|c|}{ Mittelzahlen } \\
\hline & & \multirow{2}{*}{$\mathbf{H}$} & \multirow{2}{*}{$\mathbf{X}$} & \multirow{2}{*}{$\mathbf{N}$} & \multirow{2}{*}{ NTO' } & \multirow{2}{*}{ UTO' } & \multirow{2}{*}{ RTO' } & \multirow{2}{*}{ NTO } & \multirow{2}{*}{ UTO } & \multirow{2}{*}{ RTO } & \multicolumn{2}{|c|}{$\mathrm{NTO}^{\prime}=100$} & \multirow{2}{*}{$\frac{\mathrm{NTO}^{\prime}}{\mathrm{N}}$} \\
\hline & & & & & & & & & & & UTO & RTO' & \\
\hline 10.N. & 890 & 0,4710 & 0,0135 & 11,37 & 106,42 & 63,65 & 42,77 & 67,82 & 49,57 & 18,25 & 59,81 & 40,19 & 9,36 \\
\hline 11./v. & 875 & 0,4336 & 0,0091 & 10,66 & 103,95 & 58,60 & 45,35 & 65,88 & 45,64 & 20,24 & 56,37 & 43,63 & 9,76 \\
\hline 12. $\mathrm{V}$. & 785 & 0,4797 & 0,0159 & 10,98 & 103,30 & 64,82 & 38,48 & 66,02 & 50,49 & 15,5 & 62,75 & 37,25 & 9,43 \\
\hline 13. $/$. & 990 & $0, \overline{5} 613$ & 0,0150 & 10,60 & 123,45 & 75,85 & 47,60 & 77,91 & 59,08 & 18,83 & 61,44 & 38,56 & 11,67 \\
\hline 14./N. & 940 & 0,5270 & 0,0205 & 13,06 & 118,16 & 71,22 & 46,94 & 71,44 & 55,47 & 15,97 & 60,27 & 39,73 & 9,05 \\
\hline 15./V. & 870 & $0, \check{5} 115$ & 0,0248 & 9,87 & 110,23 & 69,12 & 41,11 & 71,25 & 53,84 & 17,41 & 62,70 & 37,30 & 11,15 \\
\hline
\end{tabular}

Oxydationszahlen in der II. Verdauungsperiode.

\begin{tabular}{|c|c|c|c|c|c|c|c|c|c|c|c|c|c|}
\hline \multirow{3}{*}{ Tag } & \multirow{3}{*}{$\begin{array}{c}\text { Volum } \\
\mathrm{ccm} \\
\end{array}$} & \multicolumn{12}{|c|}{ Mittelzahlen } \\
\hline & & \multirow{2}{*}{ H } & \multirow{2}{*}{$\mathbf{X}$} & \multirow{2}{*}{$\mathbf{N}$} & \multirow{2}{*}{ NTO' } & \multirow{2}{*}{ UTO' } & \multirow{2}{*}{ RTO' } & \multirow{2}{*}{ NTO• } & \multirow{2}{*}{ UTO } & \multirow{2}{*}{ RTO } & \multicolumn{2}{|c|}{$\mathrm{NTO}^{\prime}=100$} & \multirow{2}{*}{$\frac{\text { NTO }^{\prime}}{\mathrm{N}}$} \\
\hline & & & & & & & & & & & UTO $\cdot$ & RTO' & \\
\hline 10. $\mathrm{V}$ & 620 & 0,3723 & 0,0129 & 9,67 & 82,52 & 50,31 & 32,21 & 60,01 & 39,19 & 20,82 & 60,96 & 39,04 & 8,53 \\
\hline 11. $\mathrm{N}$ & 485 & 0,3055 & 0,0119 & 8,81 & 73,43 & 41,28 & 32,15 & 43,12 & 32,15 & 10,97 & 56,08 & 43,92 & 8,33 \\
\hline 12. $N$ & 590 & 0,3741 & 0,0157 & 9,76 & 72,07 & $50, \check{5}$ & 21,52 & $\overline{5} 5,69$ & 39,39 & 16,30 & 70,14 & 29,86 & 7,38 \\
\hline 13./ $\mathrm{N}$ & 420 & 0,3510 & 0,0127 & 6,67 & 71,90 & 47,31 & 24,59 & 47,75 & 36,95 & 10,80 & 65,79 & 34,21 & 10,78 \\
\hline $14 . / V$ & 383 & 0,2757 & 0,0138 & 6,87 & 69,01 & 37,25 & 31,76 & 43,55 & 29,02 & 14,53 & $3 \mid \tilde{\jmath} 3,97$ & 46,03 & 10,04 \\
\hline 15. $/ \mathrm{N}$ & 530 & 0,3461 & 0,0206 & 8,85 & 73,22 & 46,77 & 26,45 & $\breve{5} 0,30$ & 36,44 & 13,86 & 63,87 & 36,13 & 8,27 \\
\hline
\end{tabular}

Am 7., 8. und 9. Mai stehen sowohl $\mathrm{G}$ als $\mathrm{N}$ in Stickstoffgleichgewicht und scheiden gleiche Mengen Stickstoff, Harnsäure und Restkörper ab.

Beim Uebergang in die rein animalische Nahrung fällt bei $G$ trotz der Vermehrung des assimilirbaren Eiweisses sowohl der Stickstoff als auch die Harnsäure und die Restkörper.

Dieser Abfall ist in den beiden Verdauungsperioden merklich, in der ersten etwas mehr.

Im Vergleich mit der Harnsäure fallen die Restkörper weniger.

Am nächsten Tage erholt sich bei derselben Kost die Assimilation. Der Stickstoff und die Harnsäure steigen in der 
Verdauungsperiode, die Restkörper steigen etwas in der Verdauungsperiode und fallen noch mehr in der Nachperiode. Sie steigen erst wieder in der Nachperiode, als die Stickstoffassimilation die Höhe des Gleichgewichtes überstiegen hat. Bei animalischer Kost reagirt somit bei G die Harnsäureausscheidung prompt auf die Assimilation des Stickstoffs, während die Restkörper nachziehen (umgekehrt ist es bei $\mathrm{N}$ beim Uebergang in die vegetabilische Kost).

Bei vollkommener Entziehung der vegetabilischen Nahrung und Ersetzung derselben durch rein animalische wiederholt sich das Spiel. Zuerst fällt, dann steigt der Stickstoff und ebenso fällt und steigt die Harnsäure. Die Restkörper unterliegen geringen Schwankungen. Bei vollkommener Entziehung der Nahrung überhaupt fallen Stickstoff, Harnsäure und auch die Restkörper.

Ihr niedrigster Stand gleich im ersten halben Tag nach der Nahrungsentziehung ist 13,6 RTO' gegen die normale etwa 35,0 RT0' am Vormittag und 25,0 RT0' am Nachmittag. Die höchsten Zahlen für die Restkörper bei $G$, bei rein animalischer Nahrung (weisses Fleisch, Schinken, Eier, Butter, Milch), sind somit kleiner als diejenigen bei Stickstoffgleichgewicht mit gemischter Nahrung. (Umgekehrt ist das Verhältniss bei $\mathrm{N}$ bei vegetabilischer Nahrung.) Der calorische Verlust $\frac{\mathrm{NTO}^{\prime}}{\mathrm{N}}$ bewegt sich gegen die Normale in geringen Schwankungen und fällt im Allgemeinen bei rein animalischer Kost.

$\mathrm{N}$ war am 7., 8. und 9. Mai im Stickstoffgleichgewicht mit gleicher Abscheidung des Stickstoffs, der Harnsäure und der Restkörper. Am 10. hat er die Hälfte der animalischen Nahrung in $\mathrm{N}$ und calorienäquivalente vegetabilische Kost verwandelt (Linsen, Bohnen, Brod, Lebzelten) und blieb dabei durch drei Tage.

In Folge dessen verminderte sich auch hier wie bei $G$ am ersten Tage die Resorption der Eiweisssubstanz. Stickstoff fiel an zwei nacheinander folgenden Tagen und erholte sich am dritten Trge beinahe bis zur Höhe des ursprünglichen Gleichgewichtes. 
Auf diesen Stickstoffabfall reagirte in den ersten 24 Stunden die Harnsäure gar nicht. Sie wurde in derselben Menge ausgeschieden wie am Tage vorher und erst am zweiten Tage fiel sie, um dann mit der erhöhten Stickstoffresorption zu steigen. Sie stieg dann weiter auch in der nachfolgenden Periode, obwohl Stickstoff bereits wieder fiel, mit einem Worte zeigte die Harnsäureabscheidung eine Trägheit der Reaction und das Bestreben in der eingenommenen Richtung zu beharren. ${ }^{1}$ )

Dagegen reagiren die Restkörper prompt und zwar den Stickstoffschwankungen entgegen - wenn Stickstoff fällt, steigt in der Regel RTO' - wenn Stickstoff steigt, fällt RTO' aber niemals unter die Normale.

Es können auch bei lediglich vegetabilischer Kost sowohl UT0` als RTO` gleichzeitig steigen und eine Kreisung der NTO'-Linie nur der Stickstofflinie (Stickstoff in Decigrammen) hervorrufen.

Die höchsten RTO'Zahlen werden somit bei vorwiegend oder ausschliesslich vegetabilischer Kost erreicht (siehe $\mathrm{Zu}$ sammenstellung). In der Verdauungsperiode sind diese Zahlen um 40-90\% höher als in der Nachperiode -- sie werden somit durch die Nahrungsaufnahme beeinflusst.

Der calorische Verlust steigt bei vegetabilischer Nahrung im Vergleiche mit dem normalen und noch mehr im Vergleiche mit dem Verlust bei rein animalischer Kost. Noch mehr steigt der calorische Verlust der Restkörper allein $\frac{\mathrm{RTO}^{\prime}}{\mathrm{N}}$. Derselbe ist weit grösser als bei gemischter und animalischer Kost auch dann, wenn Stickstoff gleich gross ist, wie die nachstehende Tabelle zeigt.

$$
\text { Zusammenstellung der RTO'-, N, } \frac{\mathrm{NTO}^{\prime}}{\mathrm{N}}-\text { und } \frac{\mathrm{RTO}^{\prime}}{\mathrm{N}} \text {-Zahlen. }
$$

1) Das könnte man aber auch so erklären, dass die relative Steigung der Harnsäure bei Stickstoffabfall in den ersten Tagen einer neuen Periode nichts Anders als eine Inanitionssteigung ist (siehe Bunge, Lehrbuch der physiologischen und pathologischen Chemie, 4. Aufl., S. 333). 
A. Bei gemischter Nahrung (normale Durchschnittszahlen von $G$ und N). B. Bei rein animalischer und $C$. bei rein vegetabilischer Nahrung und genügender Stickstoffabscheidung (der 12. und 14. Tag).

\begin{tabular}{|c|c|c|c|c|c|c|c|c|c|c|c|c|}
\hline & \multirow{2}{*}{\multicolumn{4}{|c|}{ In der Tagesmenge }} & \multicolumn{4}{|c|}{ In $\operatorname{der} \mathrm{I}$. } & \multicolumn{4}{|c|}{ In der II. } \\
\hline & & & & & \multicolumn{8}{|c|}{ Verdauungsperiode } \\
\hline & RTO & $\mathrm{N}$ & $\frac{\text { NTO }}{\mathrm{N}}$ & $\frac{\text { RTO }^{\prime}}{\mathrm{N}}$ & RTO & $\mathbf{N}$ & $\frac{\mathrm{NTO}^{\prime}}{\mathrm{N}}$ & $\frac{\mathrm{RTO}^{\prime}}{\mathrm{N}}$ & RTO & $\mathbf{N}$ & $\frac{\text { NTO }}{\mathrm{N}}$ & $\frac{\text { RTO }^{\prime}}{\mathrm{N}}$ \\
\hline $\begin{array}{c}\text { A } \\
\text { gemiseht }\end{array}$ & 64,2 & 21,8 & 8,5 & 2,94 & 36,0 & 12,1 & 9,1 & 2,97 & 28,2 & 9,7 & 7,7 & 2,91 \\
\hline $\begin{array}{c}\text { anima- } \\
\text { liseh }\end{array}$ & 58,1 & 24,1 & 7,1 & 2,41 & 36,3 & 13,2 & 7,6 & 2,75 & 21,8 & 10,9 & 6,45 & 2,00 \\
\hline $\begin{array}{l}\text { regeta. } \\
\text { bilisch }\end{array}$ & 69,3 & 20,3 & 8,9 & 3,41 & 42,7 & 12,0 & 9,2 & 3,56 & 26,6 & 8,3 & 8,7 & 3,20 \\
\hline
\end{tabular}

Aus diesen Verhältnissen kann man wohl den Schluss ziehen, dass ein Theil der in saurer Lösung durch Chamäleon oxydirbaren Restkörper vegetabilischer Provenienz ist. Der Rest ist jeder Nahrung gemeinschaftlich und unterliegt normal geringen Schwankungen. Ebensolchen geringen Schwankungen unterliegen auch die alkalischen RTO'-Zahlen. Sie lassen keine ausgeprägten Beziehungen zum Stickstoff und zur Harnsäure erkennen. Auf Stickstoffabfall reagiren sie erst dann, wenn derselbe unter eine bestimmte Grenze fällt.

Die RTO'-Zahlen sind in der ersten Verdauungsperiode durchschnittlich etwas höher als in der Nachperiode. Bei vegetabilischer Nahrung unterliegen sie grösseren Schwankungen als bei animalischer Kost und möglicher Weise hängen sie von dem Zustande des Darms ab.

Die geringe Empfindlichkeit dieser Zahlen erklärt sich aus den Seite 176 angedeuteten Verhältnissen, vor Allem aus der geringen Reactionsgeschwindigkeit zwischen den kleinsten Concentrationen des Ferricyankaliums und der Restkörper.

Um unsere Normalzahlen zusammenzustellen und zu 
Die Oxydationszahlen des Harns in saurer u. alkalischer Lösung. 195

zeigen, wie man mit denselben einen Krankheitsfall analysiren und beurtheilen könnte, geben wir hiermit ein Beispiel:

\begin{tabular}{|c|c|c|c|c|c|c|c|c|}
\hline & & & NTO & UTO' & RTO 1 & $100 \stackrel{\mathrm{Av}}{=}$ & uf & NTO \\
\hline & & & & & & UTO' & $\mid$ RTO' & \\
\hline \multirow{7}{*}{ 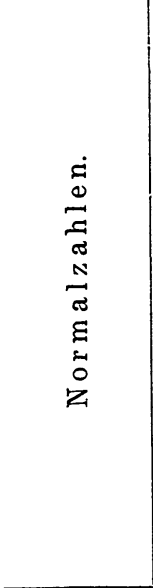 } & \multirow{4}{*}{ 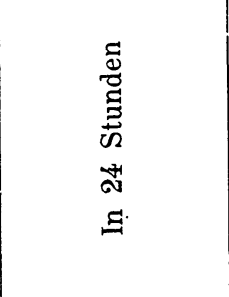 } & Mittel & 175,6 & 108,9 & 66,5 & 61,5 & 38,5 & 8,5 \\
\hline & & Die grösste Zahl & 221,1 & 143,2 & 89,3 & 51,7 & 48,3 & 9,6 \\
\hline & & Die kleinste Zahl & 134,6 & 68,3 & 51,4 & 73,0 & 27,0 & 7,83 \\
\hline & & $\begin{array}{l}\text { Die am häufigsten } \\
\text { vorkommende } \mathrm{Zahl}\end{array}$ & $175,0 \mid$ & 115,0 & 61,0 & 64,0 & 36,0 & 8,0 \\
\hline & $\begin{array}{l}\text { I. Verdauungs- } \\
\text { periode }\end{array}$ & \multirow{2}{*}{$\begin{array}{c}\text { Mittelzahlen } \\
\text { von G. und N. }\end{array}$} & 109,8 & 73,5 & 36,0 & 67,0 & 33,0 & 9,1 \\
\hline & $\begin{array}{l}\text { II. Verdauungs- } \\
\text { periode }\end{array}$ & & 75,2 & 46,1 & 28,2 & 61,3 & 38,7 & 7,7 \\
\hline & Differenz & & 35,2 & 27,4 & 7,8 & - & - & 1,4 \\
\hline $\begin{array}{l}\text { Pieber bei } \\
\text { Tabercalose } 1 \text { ) }\end{array}$ & $\begin{array}{c}\text { In } \\
24 \text { Stunden }\end{array}$ & $\begin{array}{l}\text { Einmalige } \\
\text { Benbachtung }\end{array}$ & 217,8 & 159,2 & 58,6 & 73,1 & 26,9 & 20,0 . \\
\hline $\begin{array}{c}\text { Karchexie } \\
\text { ohne Fieber2) }\end{array}$ & $\begin{array}{l}\text { In } \\
24 \text { Stunden }\end{array}$ & dto. & 123,1 & 71,8 & 41,3 & 58,3 & 41,7 & 13,4 \\
\hline \multirow[t]{4}{*}{ Nearasthenie 3 ) } & $\begin{array}{c}\text { In } \\
24 \text { Stunden }\end{array}$ & dto. & $|225,9|$ & 101,6 & 124,4 & 45,0 & 55,0 & 11,6 \\
\hline & $\begin{array}{l}\text { I. Verdauungs- } \\
\text { periode }\end{array}$ & dto. & 106,2 & 41,5 & 64,7 & 39,8 & 60,2 & 12,2 \\
\hline & $\begin{array}{l}\text { II. Verdauungs- } \\
\text { periode }\end{array}$ & dto. & 119,7 & 60,2 & 59,5 & 50,3 & 49,7 & 11,1 \\
\hline & Differenz & & $|-13, \tilde{5}|$ & $|-18,7|$ & $+5,2$ & - & - & 1,1 \\
\hline
\end{tabular}

1) 32 Jahre alte Frau. - Harn sonst normal.

2) 29 Jahre alte Frau - mit Pylorusstenose durch Neugebilde. Harn sonst normal.

3) 40 jähriger kräftiger Mann. - Spuren Eiweiss im Harne. Patient wurde wegen uratischer Diathese behandelt. 
Man sieht daraus, dass sowohl beim Fieber als auch bei der Kachexie ohne Fieber der calorische Verlust den normalen bedeutend übersteigt. Davon entfällt aber bei Fieber mehr auf die Harnsäure, deren Menge den höchsten normalen Werth übersteigt, als auf die Restkörper, die in den normalen Grenzen aber unter dem normalen Durchschnittswerth stehen. Im Verhältnisse zur Harnsäure fallen beim Fieber die Restkörper unter den kleinsten normalen Werth.

Mit Schlagworten ausgedrückt würde das heissen:

Bei Fieber: Grosser calorischer Verlust, Ueberschwemmung mit Harnsäure, wenig Restkörper.

Bei der Kachexie: Starker calorischer Verlust, hauptsächlich durch die Restkörper hervorgerufen, die aber den Körper nicht überschwemmen (nicht mehr als der höchste Normalwerth).

Bei der Neurasthenie: calorischer Verlust etwas über normal, sehr starke Ueberschwemmung durch die Restkörper. Differenzen zwischen der I. und II. Verdauungsperiode, in Bezug auf $\mathrm{N}, \mathrm{NTO}^{\prime}$ und UTO' negativ, in Bezug auf $\mathrm{RTO}^{\prime}$ positiv nicht ausreichend. Mit einem Worte eine Autointoxication durch die Restkörper.

Selbstverständlich könnte man durch Einbeziehung der alkalischen Oxydationszahlen - und durch andere Verhältnisszahlen - die Charakteristik noch viel mannigfaltiger machen.

Deshalb hoffen wir, dass bei verschiedenen Krankheiten sich Differenzen ergeben werden, die für die Beurtheilung pathologischer Zustände und therapeutischer Effecte von Werth sein werden.

\section{Die Ergebnisse.}

Die fractionirte Oxydation mit Hülfe von Indicatoren und zwar von Indigocarmin. mit Permanganat in saurer und von Alizarin mit Ferricyankalium in alkalischer Lösung lieferte neue Methoden zur Bestimmung der Reductionsfähigkeit des Harns.

Dabei wurde die Oxydirbarkeit der auf der Harnsäurestufe stehenden Körper bestimmt und die letzteren als Restkörper bezeichnet. 
Die angegebenen Methoden eignen sich auch dazu, um zu ermitteln, ob irgend ein Körper zu den Restkörpern gezählt werden kann. Auf diese Art wurde z. B. das Rhodankalium als saurer Restkörper charakterisirt.

Weiter wurden die Normalwerthe der Oxydationszahlen ermittelt und die Grenzen bestimmt, in welchen sie sich in normalen Harnen bewegen.

Es wurde in gewissen Grenzen ihre Ahhängigkeit vom Alter, von der Nahrung, vom Stoffwechsel untersucht und Beispiele für ihr Verhalten bei Krankheiten angeführt.

Weitere Folgerungen aus den angegebenen Daten, die nicht streng $\mathrm{zu}$ dem angesetzten Thema gehören, oder noch nicht genügend begründet sind, gedenken wir, nach Sammlung eines weiteren Materials, in einer besonderen Arbeit zu besprechen. 